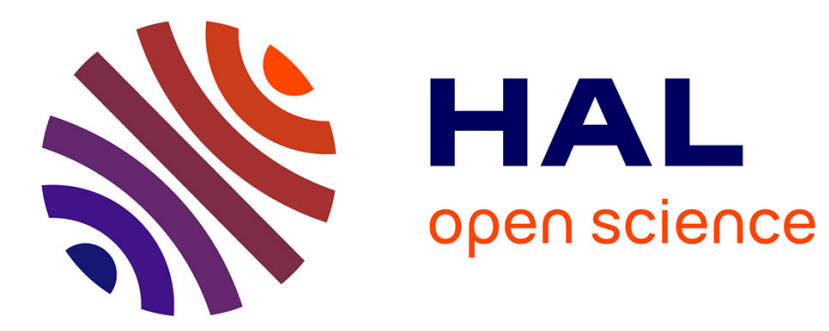

\title{
Modeling of ray paths of head waves on irregular interfaces in TOFD inspection for NDE
}

Adrien Ferrand, Michaël Darmon, Sylvain Chatillon, Marc Deschamps

\section{To cite this version:}

Adrien Ferrand, Michaël Darmon, Sylvain Chatillon, Marc Deschamps. Modeling of ray paths of head waves on irregular interfaces in TOFD inspection for NDE. Ultrasonics, 2014, 54 (7), pp.1851 - 1860. 10.1016/j.ultras.2013.12.007 . hal-01836190

\section{HAL Id: hal-01836190 https://hal.science/hal-01836190}

Submitted on 3 Jan 2019

HAL is a multi-disciplinary open access archive for the deposit and dissemination of scientific research documents, whether they are published or not. The documents may come from teaching and research institutions in France or abroad, or from public or private research centers.
L'archive ouverte pluridisciplinaire HAL, est destinée au dépôt et à la diffusion de documents scientifiques de niveau recherche, publiés ou non, émanant des établissements d'enseignement et de recherche français ou étrangers, des laboratoires publics ou privés. 


\section{Accepted Manuscript}

Modeling of ray paths of head waves on irregular interfaces in TOFD inspection for NDE

A. Ferrand, M. Darmon, S. Chatillon, M. Deschamps

PII:

S0041-624X(13)00345-4

DOI: http://dx.doi.org/10.1016/j.ultras.2013.12.007

Reference:

ULTRAS 4733

To appear in: $\quad$ Ultrasonics

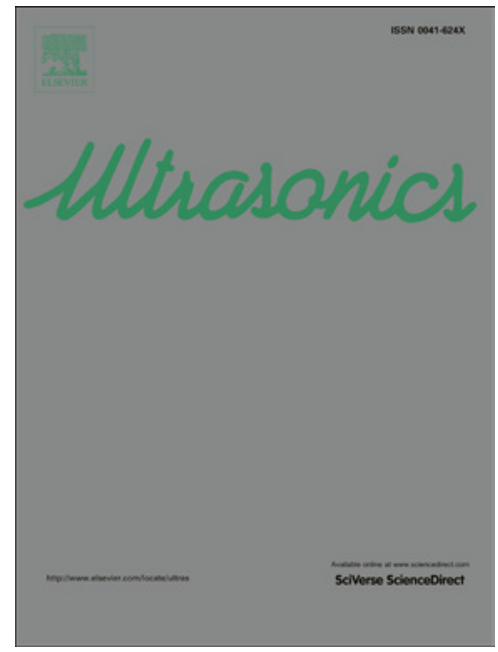

Please cite this article as: A. Ferrand, M. Darmon, S. Chatillon, M. Deschamps, Modeling of ray paths of head waves on irregular interfaces in TOFD inspection for NDE, Ultrasonics (2013), doi: http://dx.doi.org/10.1016/j.ultras. 2013.12.007

This is a PDF file of an unedited manuscript that has been accepted for publication. As a service to our customers we are providing this early version of the manuscript. The manuscript will undergo copyediting, typesetting, and review of the resulting proof before it is published in its final form. Please note that during the production process errors may be discovered which could affect the content, and all legal disclaimers that apply to the journal pertain. 
1 Modeling of ray paths of head waves on irregular interfaces in TOFD inspection for

2 NDE

3 A. Ferrand ${ }^{1}$, M. Darmon ${ }^{1}$, S. Chatillon ${ }^{1}$ and M. Deschamps ${ }^{2}$

$4 \quad{ }^{1}$ CEA, LIST, Department of Imaging \& Simulation for Nondestructive Testing, F-91191 Gif-

5 sur-Yvette, France.

$6 \quad{ }^{2}$ Univ. Bordeaux, I2M-APy, UMR 5295, 33400 Talence, France.

7

8 E-mail authors:

$9 \quad$ A. Ferrand: adrien.ferrand@ cea.fr

10 M. Darmon: michel.darmon@ cea.fr

11 S. Chatillon: sylvain.chatillon@cea.fr

12 M. Deschamps: m.deschamps@i2m.u-bordeaux1.fr

14 Corresponding author:

15 M. Darmon

16 Phone number: (+33)169082288

17 Email: michel.darmon@cea.fr

18 Postal address: CEA, LIST, Department of Imaging \& Simulation for Nondestructive Testing,

19 F-91191 Gif-sur-Yvette, France. 
Abstract:

The TOFD (Time Of Flight Diffraction) technique is a classical ultrasonic inspection method used in ultrasonic non-destructive evaluation (NDE). This inspection technique is based on an arrangement of two probes of opposite beam directions and allows a precise positioning and a quantitative evaluation of the size of cracks contained in the inspected material thanks to their edges diffraction echoes. Among the typical phenomena arising for such an arrangement, head waves, which propagate along the specimen surface and are chronologically the first waves reaching the receiver, are notably observed. Head wave propagation on planar surfaces in TOFD configurations is well known. However, realistic inspection configurations often involve components with irregular surfaces, like steel excavated specimens.

Surface irregularity is responsible for numerous effects on the scattering of bulk waves, causing the melting of surface and bulk mechanisms in the head wave propagation. In order to extend the classical ray approach on these complex cases, a generic algorithm of ray tracing between interface points (GIRT) has been designed. With respect to time of flight minimization (i.e. the generalized Fermat's principle), ray paths can be computed by GIRT for different natures of waves scattered by the complex surfaces or by flaws. The head wave fronts computed by GIRT are notably in good agreement with FEM simulated results. This algorithm, based on pure kinematic analysis of waves propagation, represents a first step in the future development of a complete ray theory for head waves simulation on irregular

40 interfaces. Keywords: head wave, ray tracing algorithm, irregular surface, wave diffraction, non-destructive evaluation 


\section{Introduction}

47

Head waves are well known phenomena occurring in seismic inspections: they correspond to the first wave received by seismic probes, giving so their names. Typical seismic inspections deal with configurations involving two media separated by a planar interface. Specific wave theories have been developed, all considering head waves on planar surfaces as critically refracted waves. These modeling approaches are based on an asymptotic integral evaluation [1], the asymptotic ray theory [2,3] or the generalized ray theory (Cagniard-De Hoop method) [4,5]. It is shown that the head wave on planar interfaces corresponds to the combination of both waves travelling along one interface side with the corresponding material sound velocity (compulsory more than that of the incident wave), and bulk waves radiated from the interface towards one material and propagating at the critical angle. The waves propagating along the interface are composed of the well-known lateral waves [1-6] and of the Goodier-Bishop type waves [7] for the case of a finite beam impacting the interface.

Head waves on planar surfaces are also observed in NDE by using specific techniques and similar configurations than the seismic ones. Among the various NDE inspections techniques, the Time of Flight Diffraction (TOFD) technique allows a precise and quantitative evaluation of surface breaking or embedded cracks. This technique uses two probes moving on the inspected material surface with a fixed Probe Center Spacing (PCS). The TOFD configuration is particularly well adapted to generate and receive head wave signals [8-10] (sometimes used for surface breaking cracks detection).

The CIVA software platform for NDE simulation, developed at CEA/LIST, enables a complete simulation of TOFD inspections: indeed different semi-analytical models $[8,11,12]$ have been integrated to model all the echoes observed in a TOFD configuration (head wave, 
flaw edges diffractions, back wall echo...). The developed model for head waves simulation is based on the asymptotic ray theory [3] which has also been used to improve the modeling of flaw corner echoes $[13,14]$. Nevertheless, the existing head wave simulation can only be applied for a planar specimen composed of an isotropic medium.

TOFD and other pitch-catch configurations are also classically used on non-planar interfaces both in NDE (inspections of cylinders, nozzles [8]..) and in the seismic domain: indeed in works carried out by Zhou and Chen [15], head wave travel times have been calculated by numerical simulations on some irregular surfaces for some seismic inspections. It has been shown that the head wave propagation is more complex on irregular surfaces than on planar surfaces. Indeed, whereas the head wave on a planar interface is due to the critical refraction of the incident wave and includes a surface wave component propagating along the planar surface (called lateral wave), the head wave propagation on irregular surfaces implies propagations in the material bulk. The received head wave signal on irregular surfaces has a different amplitude than in the planar case and a travel time compatible with the hypothesis of propagation in the material bulk. Analytical modelings of the head wave propagation around the Earth have also been proposed in several seismic studies. Indeed, using an integral representation of the solution, the received head wave signal has been evaluated for slightly curved irregularities $[16,17]$ of surface parametric equation $F(x)=x^{2} / 2 D$ with large values of the radius of curvature $D$ compared to the surface size, and for spherically symmetric radially heterogeneous media [18]. These studies confirms conclusions given in [15] concerning the time of flight and the amplitude of the head wave.

In the present study, complete interpretation and simulation of the head wave propagation mechanisms for irregular surfaces are proposed. This approach leads to the development of a generic algorithm of ray tracing between interface points (called in the following for simplification Generic Interface Ray Tracing/GIRT) which is able to compute the travelling 
front construction [22], the finite difference Eikonal equation solver [23-27] which also

path of head waves in specimens inspected by TOFD technique. Based on an adaptation to the NDE domain of seismic ray tracing algorithms, GIRT is a new method for solving the twopoints ray tracing problem, which is the calculation of the true ray between two defined points: the source and observation points.

Ray-tracing techniques are often employed for modeling wave propagation in electromagnetism, optics, seismology ... Traditional ray tracing algorithms are the shooting method [19] and the bending method [20,21]. The shooting method, well adapted for field propagation modeling, fixes the source point, takes initial ray direction and then finds the location of the observation point; in the view of two-points rays tracing, an iterative procedure for finding the starting direction can be used to yield the desired observation point. Ray bending is a variational approach which perturbs some initial estimate of the ray path, the ends being fixed, until the true ray which minimizes the travel time is found. The two previous traditional methods are heavy in time computation for solving the two-points ray tracing problem and not efficiently adapted to deal with diffracted waves and shadow areas in which head waves around irregular surfaces propagate.

To improve efficiency of the shooting method on the diffracted waves calculation, seismic ray tracing algorithms based on a grid based scheme were developed more recently : wave

114 algorithms [28-35] (also called minimum travel time tree - MTTT) which are relatively

115 underdeveloped algorithms based on Huygens' and Fermat's principles. Compared with the traditional ray tracing algorithms, the grid based scheme has several advantages for 
117 application to NDE modeling: it is able to easily locate ray paths in shadow zones; it is

118 numerically stable and can find the first arrival in complex media.

119 The current study proposes, for modeling the propagation of an incident beam towards a 120 receiving probe in a specimen of irregular surfaces including flaws, an approach based on

121 seismic SPM algorithms adapted to ultrasonic NDE and specifically designed for the two

122 points ray tracing problem. For a 2D propagation, the initial SPM Moser's algorithm [29]

123 consists in launching rays from a source, in considering in a 2D grid the adjacent node with

124 minimal travel time as a secondary source, in repeating the previous process so as to

125 propagate first arrival waves to the 2D grid nodes. More recently, Zhang et al [35] developed

126 the interface source method (ISM): as in Moser's SPM, first arrival waves still propagate

127 using a ray shooting scheme from nodes to nodes in a $2 \mathrm{D}$ grid but on the other hand only

128 interface points are taken as secondary sources. The developed GIRT model uses a new SPM

129 approach specifically devoted to two points ray tracing in a propagation medium constituted

130 of homogeneous volumes (in terms of sound velocity). The GIRT takes advantages that in the

131 latter case waves propagate in straight lines between interface points: only the specimen

132 interfaces are meshed (in a simple 1D mesh for a 2D propagation), the source (on the emitter)

133 and observation (on the receiver) points are added to the mesh and the wave ray path of

134 minimum travel time passing through mesh points is found between the two fixed points. One

135 of the major advantages of this GIRT method is the fast processing of very irregular surfaces

136 as only the surfaces of the specimen are meshed and not all the specimen volume like in other

137 SPM algorithms [28-35]. In addition, GIRT directly traces in an optimized manner (a two

138 points ray tracing) the shortest path from a source reaching any required observation point

139 located anywhere (outside the $2 \mathrm{D}$ grids needed by previous methods to propagate all shortest

140 paths to all grid nodes). 
Furthermore, compared to the ISM method [35], the developed GIRT algorithm has

142

143

144

145

146

147

notably added features to meet the specificities of ultrasonic NDT applications. Firstly, whereas ISM has been shown to deal with only the first arrival or one reflection path for $\mathrm{P}$ bulk waves, the GIRT algorithm is adapted to find not only the head wave ray path (first arrival) but also all complex later arrival waves including mode or nature conversion by adding constraints on the searched ray path, fixing one or more points through which the ray path must pass, and by defining independent types of wave propagation along each path connecting the constraint points. Secondly, the GIRT can take into account all types and natures of waves propagation occurring in NDE. Indeed the propagation is available not only for the SH head waves studied in [15] (also called TH) but also for P and SV waves (also called L and TV). Surface waves as Rayleigh waves and head waves are taken into account as well as diffractions from surfaces in any kind of waves. Finally, GIRT approach is extended to flaw scattering modeling since the flaws contained in the specimen are also meshed by the GIRT algorithm in order to model rays diffracted from flaws. Consequently the GIRT algorithm is generic for application to NDE as it can deal with any ultrasonic wave propagating near irregular surfaces or flaws of any geometry (CAD defined). Finally this tool is able to calculate valid geometric ray paths after interaction with interfaces or flaws. One interest of the study presented in this paper is also to evaluate the applicability of such a method initially based on seismic ray tracing in NDE applications for which the characteristic dimensions (propagation distance or scatterer size) to the wave length ratio can be smaller than in geophysics. The current study shows that GIRT leads to a better understanding of the head wave propagation and its interaction with the surfaces. In that aim, a data fusion of simulated results obtained by both GIRT and a FEM-based method is carried out.

The paper is organized as follows. The theoretical principles of the approach, the GIRT algorithm process and the GIRT capabilities are described in section 2; some ray tracings and 
166

167

168

169

170

171

172

wave fronts of the head wave and some later arrival waves, simulated with GIRT in 2D applications, are compared for validation with FEM results in section 3.

\section{The Generic Interface Ray Tracing (GIRT) method}

The development of a ray tracing method allows a better understanding of the head wave propagation on irregular surfaces by providing valid ray paths. The interest of such a study is described in parts 2.1 and 2.2. The developed ray tracing method (GIRT) is generic by the fact that it supports any kind of wave propagation and interaction with an irregular surface, and describes the physical phenomena involved in the head wave propagation. Its theoretical principles, functionalities and operating process are detailed in part 2.3.

\subsection{Head waves in planar surfaces configurations}

For a planar surface inspected in TOFD, a head wave is generated at the interface between the material and the emitter and propagates in the material along the surface, and is radiated as a bulk wave in the wedge, reaching the receiver. For planar surfaces, the head wave is seen as the result of a critical refraction phenomenon of the incident wave on the interface between the two media. Fig. 1 describes, in a TOFD inspection of a planar specimen containing an embedded crack, the propagation of different waves including the head wave.

Fig. 2 illustrates the B-scan measured in the inspection described in Fig. 1. Three kinds of signals can be observed and are characteristics of a TOFD inspection on a planar specimen. These signals correspond respectively to the head wave (1), the diffraction (2,2') of the refracted bulk wave on the edges of the crack and the reflection (4) of the refracted bulk wave on the specimen bottom. 


\subsection{Head wave in irregular surfaces configurations}

193

TOFD inspections of specimen with irregular entry surfaces can be required for specific NDE applications. For example, the process of repairing a material including a surface breaking crack implies to remove the matter around the defect then to replace the removed matter. The TOFD technique is applied on the specimen between the two reparation stages in order to ensure that the entire defect has been removed. The geometry of the specimen after matter removing is presented in Fig. 3.

A good understanding of the head wave propagation on such surfaces is essential for the complete modeling of TOFD inspections on realistic configurations. In particular, the head wave signal is one of the main references for the detection and the location of potential defects in the specimen. As explained in [15], irregularities of the specimen surface induce modifications on the head wave received signal compared to that observed on planar interfaces. Indeed such modifications can be noticed on experiments: the B-scan presented in Fig. 4 is extracted from a TOFD inspection measurement on the specimen described in Fig. 3 and highlights two kinds of differences which can be observed when scanning over the surface irregularities. Firstly, the head wave time of flight appears to be incoherent with the hypothesis of head wave propagation all along the entry surface: indeed the measured travel time is less than that of a surface wave propagating all along the complex surface. Secondly, the irregular surface causes an important decrease (more than $15 \mathrm{~dB}$ ) of the head wave amplitude compared to a planar interface.

To understand the physical process responsible for the observed head wave propagation on complex surfaces, an analysis of the nature of the field propagating near such surfaces has been carried out: the emitter generates a refracted field in the material which interacts with the 
surface. In particular, surface corners are responsible for diffracting the field in their geometrical shadow. Thus head wave propagation cannot be considered as a critical refraction anymore like in the case of planar surfaces, but the result of the diffractions of the generated refracted field on the surface irregularities. Fig $5 a$ describes such behavior on an example of irregular surface: the head wave, after a bulk refraction of the incident wave, is diffracted two times on the surface corners, in order to create a diffracted wave, which is then refracted again towards the receiver. In order to visualize this behavior, some numerical simulations have been carried out with the hybrid technique CIVA/ATHENA [36] using both analytical models and finite elements simulations near the surface irregularities. The principle of this technique is the following. A volume in the specimen is defined, embedding the surface and its irregularities. The field reaching the volume entry is calculated by the analytical CIVA pencil method on the volume border. Then the field is propagated in the defined volume by the finite elements scheme ATHENA. The results of this simulation are presented in Fig. $5 b$ and show that there exists a field diffracted from the surface corner in the geometrical shadow area of the irregular surface: the corresponding diffracted rays well generate the head wave (Fig $5 a$ ).

\subsection{GIRT objectives and principles: construction of valid ray paths}

$$
\text { Considering the behavior of the head wave near an irregular surface, the ray theory }
$$
formalism is then applied for the two points ray tracing problem. The head wave propagation and more generally all the waves in the specimen are then the result of specific interactions between the wave field inside or outside the specimen and the specimen surface: the ray path representing this propagation is the combination of specific rays connected to each other by secondary source points. These secondary source points are located on the specimen surface 
and correspond to the locus of each interaction responsible of the wave propagation. The rays are a representation of the wave propagation between each secondary source points.

The GIRT ray tracing formalism is thus based on the Huygens principle. Considering known source point $M_{s}$ and observation point $M_{o}$, there exists a secondary source points collection $\left\{M_{i}\right\}_{i \in(1, \ldots, \mathrm{N})}$ located on the specimen/flaws surfaces which represents the locus of all potential interactions between the wave field and the surfaces: the size $N$ of the secondary source points increases with the complexity of the surface irregularities. The ray path associated to a wave propagation between the points $M_{s}$ and $M_{o}$ is then a combination of elementary particular rays $\left(M_{j} M_{l}\right)_{(j, l) \in(s, 0,1, \ldots, N)^{2}}$ whose extremities are secondary source points $M_{i}$ among the collection $\left\{M_{i}\right\}_{i \in(1, \ldots, \mathrm{N})}$ of an elementary ray emitted from $M_{s}$ and of an elementary received on $M_{o}$.

In the following, it is illustrated on examples (Fig. 6) that this principle of secondary sources can be applied to represent all physical waves in a propagation medium containing interfaces and flaws. In a general manner, numerous waves including the head wave are emitted from a source point $M_{s}$ and received on an observation point $M_{o}$ : these waves are characterized by their interactions with the specimen surfaces involved in their propagation and then by the secondary source points constructing the associated ray path. Moreover, theses interactions may be responsible of a wave mode or wave nature conversion: consequently, each elementary ray $\left(M_{j} M_{l}\right)_{(j, l) \in(s, 0,1, \ldots, N)^{2}}$ can represent the propagation of a longitudinal (L) bulk wave, transversal bulk waves (T in an isotropic medium, qT1 and qT2 in anisotropic one), lateral surface wave (L or T) or Rayleigh-like (Rayleigh, Generalized Rayleigh, Stoneley) wave depending on the type of interaction located on the secondary source point $M_{j}$ which is the departure point of ray $\left(M_{j} M_{l}\right)_{(j, l) \in(\mathrm{s}, 0,1, \ldots, N)^{2}}$. 
Two inspection configurations involving irregular surfaces are studied as examples in Fig.

264

265

266

267

268

269

270

271

272

273

274

275

276

277

278

279

280

281

282

283

284 285

6. The first configuration (Fig. 6a) is a three propagation media one for inspection of a specimen including a corner irregularity on its entry surface, an internal interface with slopes and a defect. The case of an isotropic elastic specimen with an entry cylindrical surface is shown in Fig. 6b. The upper medium is a fluid one in both cases. Each ray path in Fig. $6 a$ and Fig. $6 b$ represents one particular wave propagating. Only some ray paths among all existing are shown as examples and the secondary source collection $\left\{M_{i}\right\}_{i \in(1, \ldots, \mathrm{N})}$ is consequently not exhaustive.

As to the configuration of Fig. $6 a$, the ray path $M_{s} M_{2} M_{5} M_{12} M_{o}$ (path 1) is the ray with the shortest time of flight and thus corresponds to the head wave propagation: its path is composed of a refraction at $M_{2}$ of the longitudinal bulk wave emitted at $M_{s}$, a corner diffraction at $M_{4}$ without mode or nature conversion and a refraction towards the observation point $M_{o}$. However the diffraction of the L bulk wave at the corner $M_{5}$ implies also a mode conversion into a $\mathrm{T}$ wave and gives after refraction at $M_{11}$ the ray path $M_{s} M_{2} M_{5} M_{11} M_{o}$ (path 2 in Fig. 6a) with a higher time of flight than the head wave ray path. The ray path $M_{s} M_{3} M_{4} M_{5} M_{7} M_{10} M_{o}$ (path 3) is associated to a L bulk wave critically refracted at $M_{3}$ into a L lateral wave which propagates along the surface and is critically reradiated at $M_{10}$ into a bulk $\mathrm{L}$ wave. As another example the secondary source point $M_{7}$ also converts the lateral wave into a Rayleigh wave which radiates at $M_{9}$ a bulk L wave and gives the ray path $M_{s} M_{3} M_{4} M_{5} M_{7} M_{9} M_{o}$ (path 4). Finally the ray path $M_{s} M_{1} M_{6} M_{8} M_{13} M_{o}$ (path 5) corresponds to a $\mathrm{L}$ bulk wave emitted at $M_{s}$ and refracted at $M_{1}$ which is reflected on the internal interface of the specimen on $M_{6}$ and diffracted on the edge defect on $M_{8}$ before reaching $M_{o}$. 
Fig. $6 b$ shows the ray paths of different waves which propagate close to a surface with a

287

288

289

290

cylindrical irregularity. The head wave propagation is associated to the shortest ray path $M_{s} M_{1} M_{4} M_{5} M_{9} M_{o}$ (path 1): the emitted L bulk wave is refracted in the specimen at $M_{1}$ and diffracted on the cylindrical irregularity in a surface creeping ray $M_{4} M_{5}$; the wave propagates along the irregularity and is then radiated again at $M_{5}$, and refracted at $M_{9}$ towards the observation point $M_{o}$. Similarly to the path 3 of Fig. $6 a$ along the corner irregularity, the ray path $2\left(M_{s} M_{2} M_{3} M_{6} M_{8} M_{o}\right)$ represents the $\mathrm{L}$ lateral wave propagation along the cylindrical irregularity generated by a critical refraction at $M_{2}$ and the critical radiation at $M_{8}$. A

Rayleigh wave is also created at $M_{6}$ which radiates at $M_{7}$ and give the path 3 $M_{s} M_{2} M_{3} M_{6} M_{7} M_{o}$

Ray paths not shown on Fig. $6 a$ and Fig. $6 b$ result from other interactions in the specimen. For example, mode conversion of the emitted L bulk wave occurs at the refraction on the entry surface, at specular reflections or at diffractions on the specimen surface irregularities or flaws (like at $M_{8}$ in Fig. $6 a$ ) and allows the propagation of several transversal bulk waves into the specimens. Mode conversions of bulk waves into Rayleigh-like waves also occurs on surface irregularities (at $M_{4}, M_{5}, \ldots$ in Fig. $6 a$ or $M_{3}$ in Fig. $6 b$ ).

Finally the purpose of the GIRT is to find all physical paths described above in isotropic or anisotropic media using the Generalized Fermat's Principle. This principle is a basic one of an asymptotic ray theory, the Geometrical Theory of Diffraction (GTD) [37] which extends Geometrical Acoustics by adding rays diffracted from canonical scatterers (edges, corners, curved smooth objects...) to the classical geometrical (refracted and reflected) rays. In the aim of ray tracing, the Generalized Fermat's Principle allows to determine if a ray path represents a physical wave propagation and is consequently valid. Indeed this principle indicates that the valid ray path for a wave resulting from particular interactions between the 
310

311

312

313

314

315

316

317

318

319

320

321

322

323

324

325

326

327

328

329

330

331

332

333

334

wave field and surfaces and is the one providing the stationary time of flight and respecting the predefined conditions imposed to the wave propagation (choice of the modes for instance). This principle is verified by finding the minimal time of flight and the associated ray path. Consequently the principle of the GIRT is to find the valid ray path respecting the Generalized Fermat's principle and achieving the wave propagation conditions using a time of flight minimization technique. Its algorithm process is described in the following section.

\subsection{GIRT algorithm process}

The steps of GIRT algorithm process are described in this section for the general case of anisotropic media and schematically represented in the functional diagram of Fig. 7. This algorithm process is the subject of a patent filing [38].

This process is first described when used for determination of the head wave path, the first arrival (like the paths 1 on Fig. $6 a$ and Fig. $6 b$ ) and is called in that case GIRT-A. The input data (step 1) of the GIRT are the CAO description of the specimen in which the ray path has to be calculated, the source and observation points which are the ray extremity points, the slowness surfaces for the wave propagation mode desired inside and outside the specimen, the description of defects inside the specimen and the discretization fineness of the specimen or flaw surfaces which defines the calculation accuracy.

Indeed the next steps of the algorithm process is the discretization of both each specimen surface (step 2) and of each existing defect (step 3) in order to deal with any irregular surface: for the configuration of Fig. $6 a$, the two irregular surfaces and the planar defect are meshed. The obtained discretization points collection $\left\{M_{i}\right\}_{i \in(1, \ldots, \mathrm{N})}$ constitutes the secondary sources collection for waves diffraction as stated by the Huygens principle in a propagation medium constituted of homogeneous volumes. Consequently these secondary sources are possible $M_{i}$ 
335

336

337

338

339

340

341

342

points composing the ray path as described in section 2.3. The GIRT at this step 2 uses a similar approach to the interface source method (ISM) developed by Zhang and al [35] since interface points are seen as secondary sources.. On the other hand, the discretization carried out by GIRT differs from that performed by ISM [35] and other SPM algorithms [29]. Indeed as only the surfaces and the defects are discretized, the ray path searching is done only through secondary sources located along the specimen and defects surfaces and not trough a grid covering all the specimen volume. The obtained secondary sources collection has a limited size $N$ compared to that generated from a volume discretization and provide a more stable and faster research of ray paths.

To determine the valid secondary sources involved in the searched ray path in the view of the two points ray tracing problem, the elementary paths between two secondary sources are calculated (step 4): an elementary path represents the wave propagation along a direct path involving two and only two $M_{i}$ secondary sources without any other diffraction effect. All the valid elementary paths between two $M_{i}$ points respecting the previous condition are calculated for all the $M_{i}$ points and the associated elementary times of flight are deducted from the slowness surfaces given and stored by the algorithm process (step 5). As an example, the ray $M_{2} M_{5}$ in Fig. $6 b$ is an elementary ray path of the path 1 and path 2.

A secondary sources collection, an elementary paths collection and an associated elementary times of flight collection have been obtained during the previous steps. The $M_{s}$ source and $M_{o}$ observation points are added in step 6 to the existing secondary sources collection and all the new valid elementary paths involving one of these two points are calculated and taken into account in the collections. The interest of having separated this step 6 from step 5 is to allow the ray path calculation for different couples of source and observation points but with the same specimen without needing to recalculate at each ray 
359

360

361

362

363

364

365

366

367

368

369

370

371

372

373

374

375

376

377

378

379

380

381

382

383

calculation all the other elementary paths in the specimen (already independently determined at step 5).

Finally, knowing the secondary sources collection and the elementary paths collection, the search for the valid wave ray path in the view of the two point ray tracing reduces to an optimization problem. It indeed corresponds to the determination of which secondary sources $M_{i}$ are involved in the ray path and then which elementary rays $\left(M_{j} M_{l}\right)_{(j, l) \in(1, \ldots N)^{2}}$ compose the ray path associated to the minimal time of flight. This approach is called the Shortest Path Method (SPM) [29] in the seismic domain and is applied in the GIRT (step 7). The secondary source points collection are the nodes of an oriented graph. In this graph, two different vertices are connected by an elementary ray path included in the collection. At each path is associated a cost which is the elementary time of flight between of the path. Once the graph is obtained, the optimal route (leading to the shortest time of flight between the source and observation points) is obtained by the classical Dijkstra algorithm [39], which has the major advantage to be a fast route search algorithm since all possible routes are not calculated: only the most likely optimal routes are taken into account. At that step, the GIRT algorithm differs strongly from the ISM developed by Zhang and al [35]. ISM is conceived to perform ray shooting in a grid meshing all the volume by launching rays from a source in all directions and by propagating first arrival waves from nodes to nodes in the grid: consequently a ray path reaching a predefined observation point cannot be found directly notably if this point is not a grid node. In GIRT, the application of the Dijkstra algorithm on the secondary sources collection is well fitted and specific of the two point ray tracing problem. Indeed, the source and observation points have been integrated in the secondary sources collection so that the fast route search gives directly the valid ray path between these two points.

$$
\text { Later arrival waves (for example all paths represented in Fig. } 6 a \text { and Fig. } 6 b \text { except the }
$$
paths 1) can also be modeled by a GIRT-B version by adding constraints on the searched ray 
384

385

386

387

388

389

390

391

392

393

394

395

396

397

398

399

400

401

402

403

404

405

406

407

408

path, fixing one or more points through which the ray path must pass. In the case of a ray path calculation with obligatory points of passage, the GIRT-B process differs from that previously described for the head wave ray path (GIRT-A process) only by some slight changes. First, the GIRT-B inputs include in addition the list of sorted constraint points and the definition of independent types of wave propagation along each path connecting these points. Then the GIRT-B operates as follows: a couple of points is formed by the source point and the first constraint point, then several other couples by two adjacent constraint points and the last couple by the last constraint point and the observation point. An optimized ray is then searched between the two points of each couple: in that aim, the previous points are seen as the source and observation points for GIRT-A process and the GIRT-A process is consequently carried out independently for each couple, the latter having its own type of propagation, allowing all type of wave propagation in the specimen. At last, all the calculated couples ray paths are concatenated to obtain the complete ray path between the source and observation points.

\subsection{GIRT capabilities}

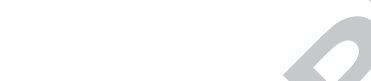

GIRT is thus a generic algorithm calculating geometrical paths and the corresponding ray time of flight propagating in isotropic or anisotropic media. It has been designed to determine the valid ray path between two points assuming one or several given interactions between the current ray and the irregular surface. The two points can be located anywhere inside or outside the specimen.

Two kinds of interactions, accounted by GTD [37], are supported by GIRT: diffraction on smooth objects, leading to surface creeping rays (see the ray $M_{5} M_{6}$ on Fig. 6 b), and diffraction on wedges or edges (see Fig. 6a), leading to bulk or surface diffracted rays. 
409

410

411

412

413

414

Furthermore, the propagation of numerous wave modes is taken into account with potential mode conversion at the surface: the longitudinal and transversal modes (possibly occurring for different kinds of waves: bulk waves, lateral waves, reradiated head waves or surface waves...), and the surface Rayleigh-like waves.

All the ray paths presented on Fig. $6 a$ and Fig. $6 b$ are modeled by setting some wave propagation conditions supported by the GIRT. As an example the head wave ray paths 1 of Fig. $6 a$ and Fig. $6 b$ are calculated using by finding the shortest ray path for a longitudinal bulk wave without mode conversion. The ray path 2 of Fig. $6 a$ is found for the condition of a wave propagation with the shortest time of flight and a mode conversion from $\mathrm{L}$ bulk to $\mathrm{T}$ bulk on the surface irregularity. The ray path 3 of Fig. $6 a$ and the ray path 2 of Fig. $6 b$ are modeled imposing a surface propagation condition (detailed in the comments of Fig. 14) to the ray path in order to propagate lateral waves along the specimen surface. Similarly the ray path 4 of Fig. $6 a$ and ray path 3 of Fig. $6 b$ are found using the same surface propagation condition and that of a wave mode conversion from a lateral wave to a Rayleigh wave. Finally the ray path 5 of Fig. $6 a$ is calculated using a reflection condition on the internal surface and an obligatory point of passage $M_{8}$ on the defect edge.

Thanks to the previously described capabilities, all the interactions occurring in the case of an irregular surface and presented in this section are modeled by GIRT. In next Part 3 of this paper, GIRT is applied to the head wave path modeling in TOFD near irregular surfaces and only the waves which may be responsible of the head wave propagation or examples of the least later arrival waves are studied. It is shown in Part 3 that GIRT provides accurate predictions for the time of flight and wave fronts of both the head wave and even later propagation waves in TOFD near irregular surfaces. 


\section{GIRT simulated results and theoretical validations}

434

435

436

437

438

439

440

441

442

443

444

445

446

447

Calculations using GIRT of ray paths and wave fronts are shown in this section in the aim of modeling the propagation of head waves near irregular surfaces in some TOFD configurations. Such simulations are compared and superimposed to FEM ones.

\subsection{Validation of the head wave time of flight}

A theoretical validation of GIRT is performed by comparing times of flight calculated with both GIRT and finite-elements (FEM) simulations obtained with CIVA/Athena, which is considered here as a reference model. The comparison deals with TOFD inspection simulations for several inspection directions $\left(\theta=45^{\circ}, 60^{\circ}\right.$ and $75^{\circ}$ where $\theta$ is the angle defined in Fig. 8) with $\mathrm{L}$ waves emitted at $5 \mathrm{MHz}$. The specimen is a planar stainless steel block which is inspected using two contact planar rectangular transducers $(6 \mathrm{~mm} * 5 \mathrm{~mm})$. The specimen entry surface has two slopes. The head wave path determined by GIRT is illustrated in blue in Fig. 8 and includes a diffraction on the surface corner.

The times of flight obtained by GIRT have been compared to the results of the FEM simulation software for different slope angles and are shown in Fig. 9. Each graph of the Fig. 9 is associated to a particular inspection direction (from $\mathrm{L} 45^{\circ}$ to $\mathrm{L} 75^{\circ}$ ), the solid lines are the results obtained with the GIRT and the dashed lines are the results obtained by FEM simulations.

The graphs show a very good concordance between the results of the GIRT and FEM simulation, for each direction of inspection and any slope angle. The GIRT is then able to calculate the head wave time of flight with a very good precision (error less than $0.1 \%$ ). The simulated times of flight of the head wave signal received by the probe are almost 
independent of the inspection directions because the head wave signal is generated by only some refracted rays included in the beam radiated in the specimen by the emitter surface, those rays being close to the mean head wave path shown in blue in Fig. 8 including a diffraction on the corner.

\subsection{Analysis of the propagation of waves in the surface shadow}

Additional finite-element simulations on CIVA/Athena have been performed on irregular surfaces including curved parts, and the propagation wave field in the material at different times is also visualized on FEM snapshots. In this section, GIRT has been employed in the aim of identifying the nature of waves propagation corresponding to some observed wavefronts in the FEM snapshots.

Indeed, when studying the interaction on a beam with an irregular surface, numerous wave fronts are generated. For instance Fig. 10 shows FEM simulation of the waves propagation near a curved valley constituted of two curved edges separated by a planar part. Numerous wave fronts are observed in the FEM snapshot of Fig. 10, which highlights the great number of interactions occurring between the refracted field and the specimen. The nature of some of these wave fronts (numerated in Fig. 10) can be easily guessed. The surrounded front L1 corresponds to the direct L-wave refracted in the specimen by the emitter. The front L1 is reflected by the specimen bottom (dashed black horizontal line in Fig. 10) creating the Lwave front $\mathrm{L} 2$ and $\mathrm{T}$-wave front $\mathrm{T} 1 . \mathrm{T} 2$ refers to $\mathrm{T}$-waves refracted in the specimen. Other wave fronts are of more complex nature. It is consequently of interest to identify the nature of rays which have generated the observed wave fronts in the FEM simulations.

So as to identify the nature of one particular FEM wave front, the following methodology is chosen: 
- The studied wave front is identified on the FEM snapshot as the local maximum of the displacement field.

- An appropriate hypothesis on the wave propagation and interactions with the surface is formulated by the GIRT user to explain the generation of the studied wave front. Considering the previously chosen path hypothesis, GIRT builds rays until their time of flight reach the FEM snapshot time.

- $\quad$ The resulting wave front simulated by GIRT is then drawn for the FEM snapshot time by tracing the curve orthogonal to every ray found at this time of flight [40]. The GIRT simulated wave front is superimposed to the FEM snapshot and its location compared to that of the FEM studied wave front to validate the chosen propagation hypothesis.

In the present study, identification of wave fronts nature by comparing FEM and GIRT simulations is done only for wave fronts relevant for studying head waves propagation. But a similar analysis could be performed for all the other wave fronts observed on FEM snapshots.

The method is thus applied for the following wave fronts:

- The front of the refracted L-wave (Fig. 10), whose diffraction on the second curved part of the surface specimen is responsible of head wave propagation towards the receiver (as said in section 2.2).

- The L head wave (Fig. 11 and 13), received at first on the receiver.

- The $\mathrm{T}$ wave received just after the $\mathrm{L}$ head wave on the receiver.

For each figure, the ray paths (white lines) and the front (dashed black line) simulated by GIRT are superimposed to the studied FEM snapshot (in color code).

In Fig. 10, ray paths simulated by GIRT are obtained considering the hypothesis injected in GIRT of a diffraction of the refracted L-wave field on a curved object without mode conversion and without constraint point. Ray paths are thus built with the GIRT-A algorithm between one source point (emitter center) and a set of observation points located in the 
508

509

510

511

512

513

514

515

516

517

518

519

520

521

522

523

524

525

526

527 528 different hypotheses detailed hereafter are inputted in GIRT concerning the second refraction 529 (at the interface specimen/wedge of the receiver) and give the results of respective Fig. 11 and

531

532

specimen bulk after the second curved part at right, inside the shadow areas formed by the surface irregularity. The GIRT objective is here to determine between the source and each observation point the shortest path which corresponds to the head wave path. The calculated ray path indicates that the emitted wave is refracted in the specimen bulk, before being diffracted by the first curved object at left. The GIRT modeling of the diffracted field by near this curved part reveals creeping rays. Indeed, the refracted longitudinal rays reaching tangentially the first curved object are converted into creeping rays. These creeping rays are then diffracted into both bulk rays along their curved propagation and a surface ray which propagates along the planar surface. The surface ray is converted again at the junction with the second curved part into a creeping ray which is diffracted into bulk rays generated tangentially to the second curved object at right inside the shadow area caused by the surface irregularity. As seen in Fig. 10, the calculated wave front is the result of the wave diffractions on the two curved objects. The previously described head wave propagation is validated by comparing the GIRT and FEM simulated wave fronts and observing a good agreement between them.

The following two cases, presented in Fig 11 and Fig 12 are obtained with the same configuration as in Fig. 10, but the propagation of waves is extended up to the receiver, allowing observing the wave fronts received in experimental studies. As in Fig. 10, the refracted longitudinal wave is diffracted by the curved parts at left and at right and refracted again at the last surface between the specimen and the wedge of the receiving probe. Two in Fig. 12. In Fig. 11, a second refraction of longitudinal waves without mode conversion is assumed and GIRT-A is used to calculate the shortest rays between the source an observation points located on the receiver crystal surface. In Fig. 12, a second refraction with conversion 
533

534

535

536

537

538

539

540

541

542

543

544

545

546

547

548

549

550

551

552

553

554

555

556

557

of the $\mathrm{L}$ wave into a $\mathrm{T}$ wave is supposed. To apply the last hypothesis, a constraint point is defined on the plane part of the irregularity and the GIRT-B algorithm is used so as to calculate the rays using the following assumptions: when the path takes place inside the specimen, it is always travelled at the celerity of the L wave; when the path takes place both outside the specimen and from the source point to the constraint point (i.e. in the emitter wedge), it is travelled at the celerity of the $\mathrm{L}$ wave; when the path takes place both outside the specimen and from the constraint point to the receiver point (i.e. in the receiver wedge), it is travelled at the celerity of the $\mathrm{T}$ wave. By this way ray paths calculated by the GIRT-B satisfy the mode conversion L-T condition on the surface near the receiver. As in Fig. 10 and for the two cases in Fig. 11 and Fig. 12, wave fronts calculated by GIRT perfectly match with two different wave fronts simulated by FEM. Furthermore the wave of type T (Fig. 12) is received by the receiver later than that of type L (Fig. 11) as shown by comparing the location of their corresponding wave fronts for a fixed time of flight. Consequently, in the previous configuration, the head wave observed at the receiver which is by definition the first received wave logically mainly corresponds to a complete path without mode conversion involving only faster L waves. All the other FEM simulated wave fronts obtained in the snapshot of Fig. 11 lead to later arrival times if they reach the receiver.

To emphasize this conclusion about the nature of the head wave propagation, the GIRT simulated rays of a L-surface wave propagation all along the irregular specimen surface without any specimen bulk propagation hypothesis are shown in Fig. 13. To satisfy the previous condition, two constraints points are specified to the GIRT-B algorithm and are positioned on the specimen surface respectively just before and just after the surface irregularity, so as to impose the propagation of rays along the irregular surface. In that case, the surface ray is generated by critical incidence on a planar interface as described in Fig. 1 . The corresponding extracted wave front does not match the head wave front obtained from 
558

559

560

561

562

563

564

565

566

567

568

569

570

571

572

573

574

575

576

577

578

579

580

581

582

FEM simulations and confirms that the bulk mechanism stated previously for the head wave propagation is likely to predominate for very corrugated surfaces.

The results in the previous comparisons between GIRT and FEM show that the head wave propagation in the bulk implied by complex interactions between the wave and the surface must be taken into account to correctly model the head wave propagation on irregular surfaces. These comparisons between the developed ray tracing model (GIRT) and FEM simulations validate the choice of the GTD approach used in GIRT to model the complex interactions between an irregular surface and the waves radiated by the probe. The GIRT based on GTD principles is able to compute valid ray paths providing wave fronts in agreement with FEM simulated results in terms of both location and shape.

\section{Conclusion}

The modeling of TOFD configurations used in ultrasonic NDT requires the study of the head wave propagation. While analytical theories for head waves on planar interfaces are well established, the complete simulation of head waves on irregular surfaces has to be developed. In that aim, one suitable approach is to use the Geometrical Theory of Diffraction.

A generic algorithm of ray tracing between interface points (GIRT) has thus been developed using one GTD principle: the Generalized Fermat's Principle. GIRT is a specific and generic adaptation of seismic ray tracings to NDE modeling of waves propagation and to the two points ray tracing problem solving: a fast processing of the waves propagation near very irregular surfaces between two fixed points is obtained by meshing only those surfaces and the defects rather than all the volume of the propagation media like in existing seismic ray tracings. The approach is adequate for a propagation medium constituted of homogenous or weakly inhomogeneous volumes: in the latter case, the wave propagation can be modeled by 
583

584

585

586

587

588

589

590

591

592

593

594

595

596

597

598

599

600

601

602

603

604

605

606

607

608

609

610

611

612

613

tracing straight lines in a mean homogenous volume and adding travel time fluctuations [41].

The advantages of the GIRT are the specific treatment of diffracted and creeping rays in shadow areas, the account of both any irregular surfaces of the specimen and embedded defects (both CAD defined), the wave mode conversions at interfaces or flaws, and the

calculation of all waves (bulk, surface and head waves) propagating towards a predefined observation point during a TOFD inspection for NDE including both first arrival (head wave) and latter arrival waves.

By modeling the complex wave interactions with the surfaces thanks to this ray approach, GIRT is able to correctly predict the head wave time of flight and to interpret the complex waves propagation at the vicinity of an irregular interface. For very corrugated surfaces, a bulk propagation mechanism has to be taken into account for the head wave modeling. Valid geometrical rays paths are henceforth determined for head waves using GIRT pure kinematic analysis. Use of GTD for simulation of the head wave amplitude will be investigated thereafter by modeling surface creeping rays and corner diffractions. GIRT could then lead in the future to a complete modeling of the head wave propagation on irregular interfaces.

\section{References}

[1] L.M. Brekhovskikh, Waves in layered media, Academic press New York, 1960.

[2] K. Friedrichs, J.B. Keller, Geometrical acoustics. II. Diffraction, reflection, and refraction of a weak spherical or cylindrical shock at a plane interface, J. Appl. Phys. 26 (1955) 961-966.

[3] V. Červený, R. Ravindra, Theory of seismic head waves, University of Toronto Press (Toronto), 1971.

[4] L. Cagniard, E.A. Flinn, C.H. Dix, W.G. Mayer, Reflection and Refraction of Progressive Seismic Waves, Phys. Today. 16 (1963) 64.

[5] A. De Hoop, A modification of Cagniard's method for solving seismic pulse problems, Appl. Sci. Res. Sect. B. 8 (1960) 349-356.

[6] H. Uberall, Surface waves in acoustics, in Physical Acoustics 10 (W. P. Mason

and R. N. Thurston, eds.), Academic Press, New York (1973) pp. 1-60. 
[7] J. Goodier, R. Bishop, A note on critical reflections of elastic waves at free surfaces, J. Appl. Phys. 23 (1952) 124-126.

[8] S. Mahaut, M. Darmon, P. Benoist, P. Calmon, TOFD inspection simulation using Civa software, in: Proc. BINDT, 2007.

[9] S. Chaffaï, M. Darmon, S. Mahaut, R. Menand, Simulation Tools for TOFD Inspection in CIVA Software, in: Proc. 6th ICNDE, 2007.

[10] Assessment, Management of ageing of major nuclear power plant components important to safety primary piping in PWRs, Vienna: IAEA, 2003.

[11] M. Darmon, S. Chatillon, S. Mahaut, L. Fradkin, A. Gautesen, Simulation of disoriented flaws in a TOFD technique configuration using GTD approach, in: AIP Conf. Proc., 2008: p. 155.

[12] V. Zernov, L. Fradkin, M. Darmon, A refinement of the Kirchhoff approximation to the scattered elastic fields, Ultrasonics. 52 (2012) 830-835.

[13] G. Huet, M. Darmon, A. Lhémery, S. Mahaut, Modelling of corner echo ultrasonic inspection with bulk and creeping waves, in: Ultrason. Wave Propag. Non Homog. Media, Springer, 2009: pp. 217-226.

[14] S. Mahaut, G. Huet, M. Darmon, Modeling of corner echo in UT inspection combining bulk and head waves effect, in: AIP Conf. Proc., 2009: p. 73.

[15] H. Zhou, X. Chen, Ray path of head waves with irregular interfaces, Appl. Geophys. 7 (2010) 66-73.

[16] I. Lerche, N. Hill, A mean-field solution of the reflection of a spherical acoustic wave from a rough interface, J. Math. Phys. 26 (1985) 1420.

[17] I. Lerche, On the reflection of acoustic waves from a slightly curved interface, J. Acoust. Soc. Am. 81 (1987) 611.

[18] D.P. Hill, Critically refracted waves in a spherically symmetric radially heterogeneous earth model, Geophys. J. Int. 34 (1973) 149-177.

[19] B. Julian, D. Gubbins, Three-dimensional seismic ray tracing, J Geophys. 43 (1977) 95114.

[20] Y. Wang, G.A. Houseman, Tomographic inversion of reflection seismic amplitude data for velocity variation, Geophys. J. Int. 123 (1995) 355-372.

[21] Y. Wang, Seismic amplitude inversion in reflection tomography, Pergamon, 2003.

[22] V. Vinje, E. Iversen, H. Gjøystdal, Traveltime and amplitude estimation using wavefront construction, Geophysics. 58 (1993) 1157-1166.

[23] J. Vidale, Finite-difference calculation of travel times, Bull. Seismol. Soc. Am. 78 (1988) 2062-2076.

[24] J.E. Vidale, Finite-difference calculation of traveltimes in three dimensions, Geophysics. 55 (1990) 521-526.

[25] K. Koketsu, others, Finite difference traveltime calculation for head waves travelling along an irregular interface, Geophys. J. Int. 143 (2000) 729-734.

[26] N. Rawlinson, M. Sambridge, Multiple reflection and transmission phases in complex layered media using a multistage fast marching method, Geophysics. 69 (2004) 13381350.

[27] M. De Kool, N. Rawlinson, M. Sambridge, A practical grid-based method for tracking multiple refraction and reflection phases in three-dimensional heterogeneous media, Geophys. J. Int. 167 (2006) 253-270.

[28] I. Nakanishi, K. Yamaguchi, A numerical experiment on nonlinear image reconstruction from first-arrival times for two-dimensional island arc structure, J. Phys. Earth. 34 (1986) 195-201.

[29] T. Moser, Shortest path calculation of seismic rays, Geophysics. 56 (1991) 59-67. 
663

664

665

666

667

668

669

670

671

672

673

674

675

676

677

678

679

680

681

682

683

684

685

686

687

688

689

690

691

692

693

694

695

696

697

698

699

700

[30] S. Cao, S. Greenhalgh, Calculation of the seismic first-break time field and its ray path distribution using a minimum traveltime tree algorithm, Geophys. J. Int. 114 (1993) 593-600.

[31] H.J. Van Avendonk, A.J. Harding, J.A. Orcutt, W.S. Holbrook, Hybrid shortest path and ray bending method for traveltime and raypath calculations, Geophysics. 66 (2001) 648653.

[32] A. Zhao, Z. Zhang, J. Teng, Minimum travel time tree algorithm for seismic ray tracing: improvement in efficiency, J. Geophys. Eng. 1 (2004) 245.

[33] C. Bai, S. Greenhalgh, B. Zhou, 3D ray tracing using a modified shortest-path method, Geophysics. 72 (2007) T27-T36.

[34] C. Bai, G. Huang, R. Zhao, 2-D/3-D irregular shortest-path ray tracing for multiple arrivals and its applications, Geophys. J. Int. 183 (2010) 1596-1612.

[35] M.-G. Zhang, Y.-G. Jia, M.-Y. Wang, X.-F. Li, A global minimum traveltime ray tracing algorithm of wavefront expanding with interface points as secondary sources, Chin. J. Geophys. 49 (2006) 1046-1053.

[36] N. Gengembre, A. Lhémery, R. Omote, T. Fouquet, A. Schumm, A semi-analytic-FEM hybrid model for simulating UT configurations involving complicated interactions of waves with defects, in: AIP Conf. Proc., 2004: p. 74.

[37] J.B. Keller, Geometrical theory of diffraction, JOSA. 52 (1962) 116-130.

[38] Patent filing related to GIRT in progress. Its precise reference will be given in the proofs.

[39] E.W. Dijkstra, A note on two problems in connexion with graphs, Numer. Math. 1 (1959) 269-271.

[40] D. Bouche, F. Molinet, R. Mittra, Asymptotic methods in electromagnetics, Springer, 1997.

[41] B. Lu, M. Darmon, C. Potel, Stochastic simulation of the high-frequency wave propagation in a random medium, J. Appl. Phys. 112 (2012) 054902-054902.

\section{Figure Captions}

Figure 1: A NDT inspection using TOFD technique

Figure 2: Experimental B-scan observed in the TOFD inspection of Fig. 1

Figure 3: An irregular geometry studied by TOFD technique

Figure 4: TOFD inspection measurement on the specimen including a valley of finite extension as described in Fig. 3. a): experimental B-scan obtained when scanning the specimen in the extension direction (perpendicular to planes of Fig. 4b) and 4c). For the first left scanning positions, the probes are on a planar part (Fig. 4b) and they are then located on both sides of the valley (Fig. 4c). 
Figure 5: Propagation of a HW on an irregular surface (valley consisting in several planar slopes). a) Theoretical propagation of the head wave. b) Snapshot of the field calculated by FEM at the location of the diffraction phenomena (in the yellow area of Fig 5a).

Figure 6: Various paths (color lines) resulting from several interactions with two kinds of surfaces (black lines): a surface with a corner irregularity (a) and a surface with a cylindrical irregularity (b).

Figure 7: Functional diagram of the GIRT algorithm process

Figure 8: TOFD inspection used for the theoretical validation of GIRT.

Figure 9: Comparison of times of flight calculated both with GIRT and with FEM simulations (Civa/Athena) versus slope angles for different inspection directions (a: $45^{\circ}, \mathrm{b}: 60^{\circ}, \mathrm{c}$ : $\left.75^{\circ}\right)$

Figure 10: Diffraction of L-waves on curved objects. Head wave ray paths calculated by GIRT are represented in white and forms the simulated wave front in black. FEM simulated snapshot is in color code.

Figure 11: Head wave ( $\mathrm{L}$ wave) received by the transducer. At right: zoom on the receiver.

Figure 12: Hypothesis of a T wave received by the transducer. At right: zoom on the receiver.

Figure 13: Hypothesis of a $\mathrm{L}$ wave received by the transducer without propagation in the specimen bulk. At right: zoom on the receiver. 


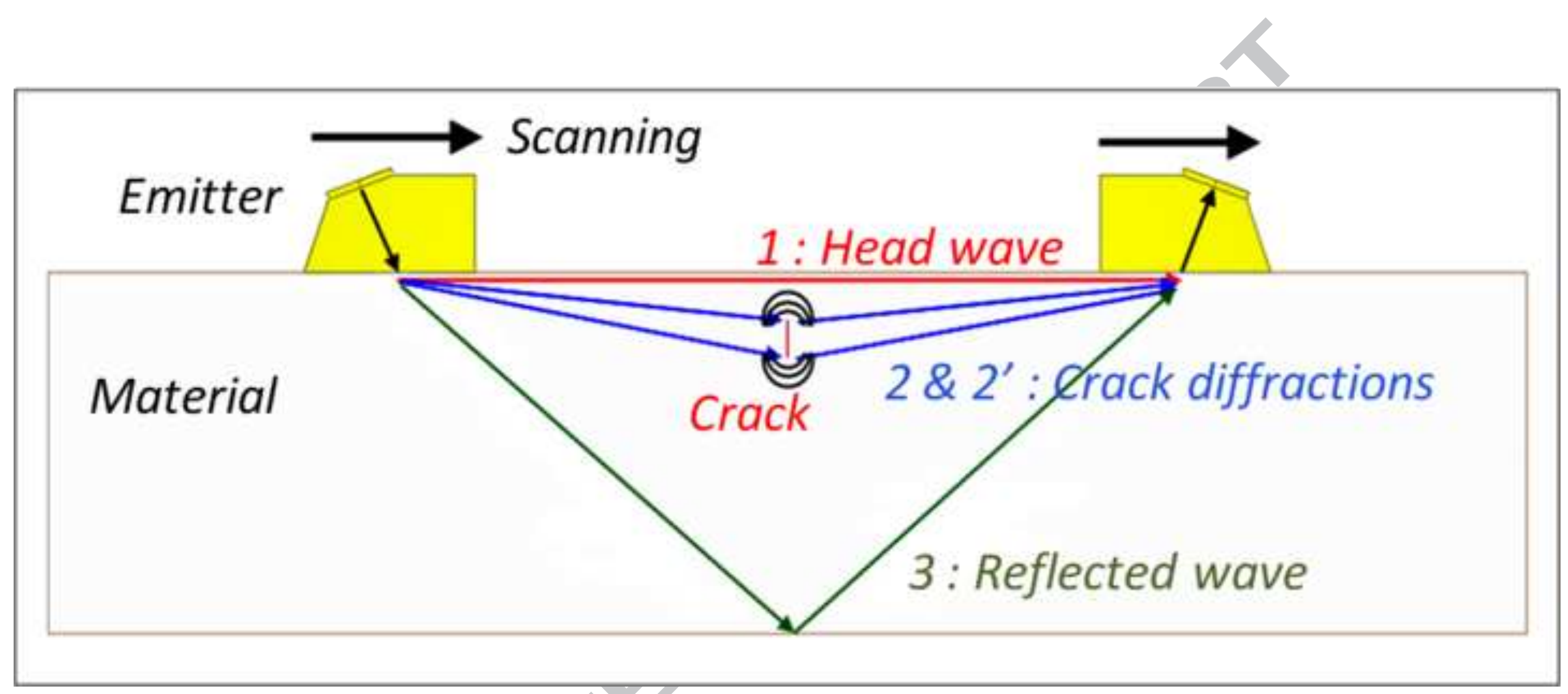




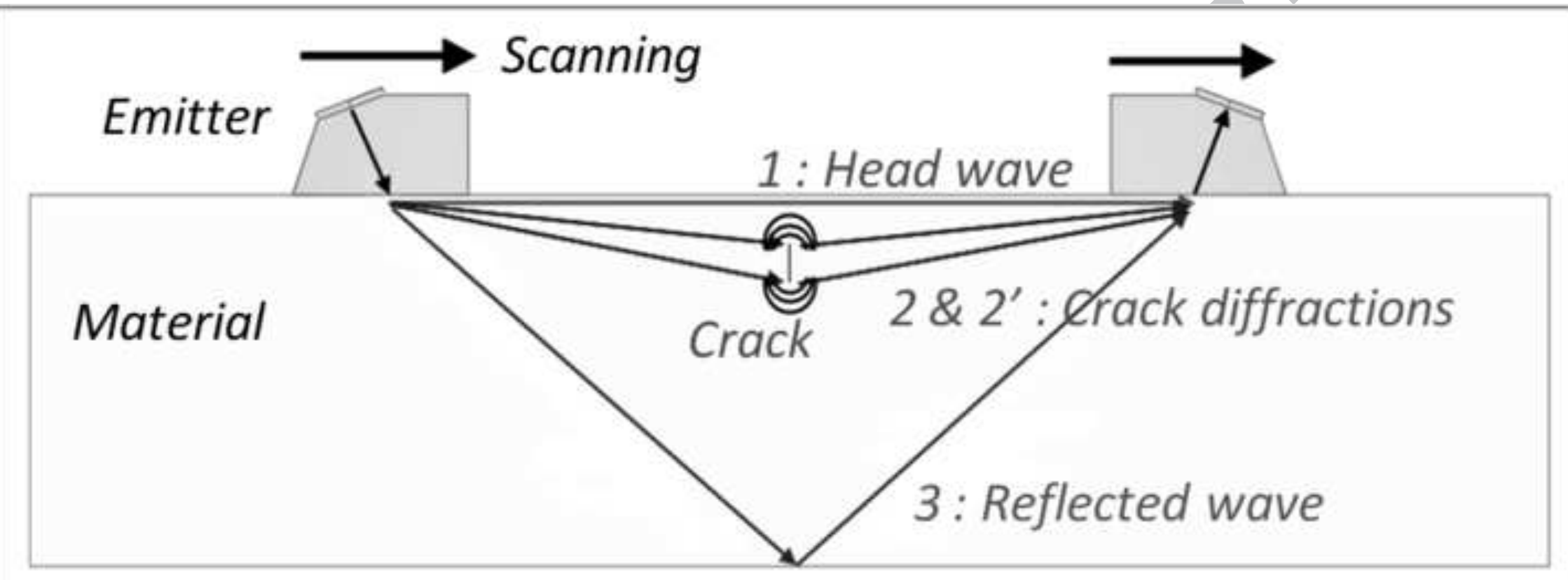




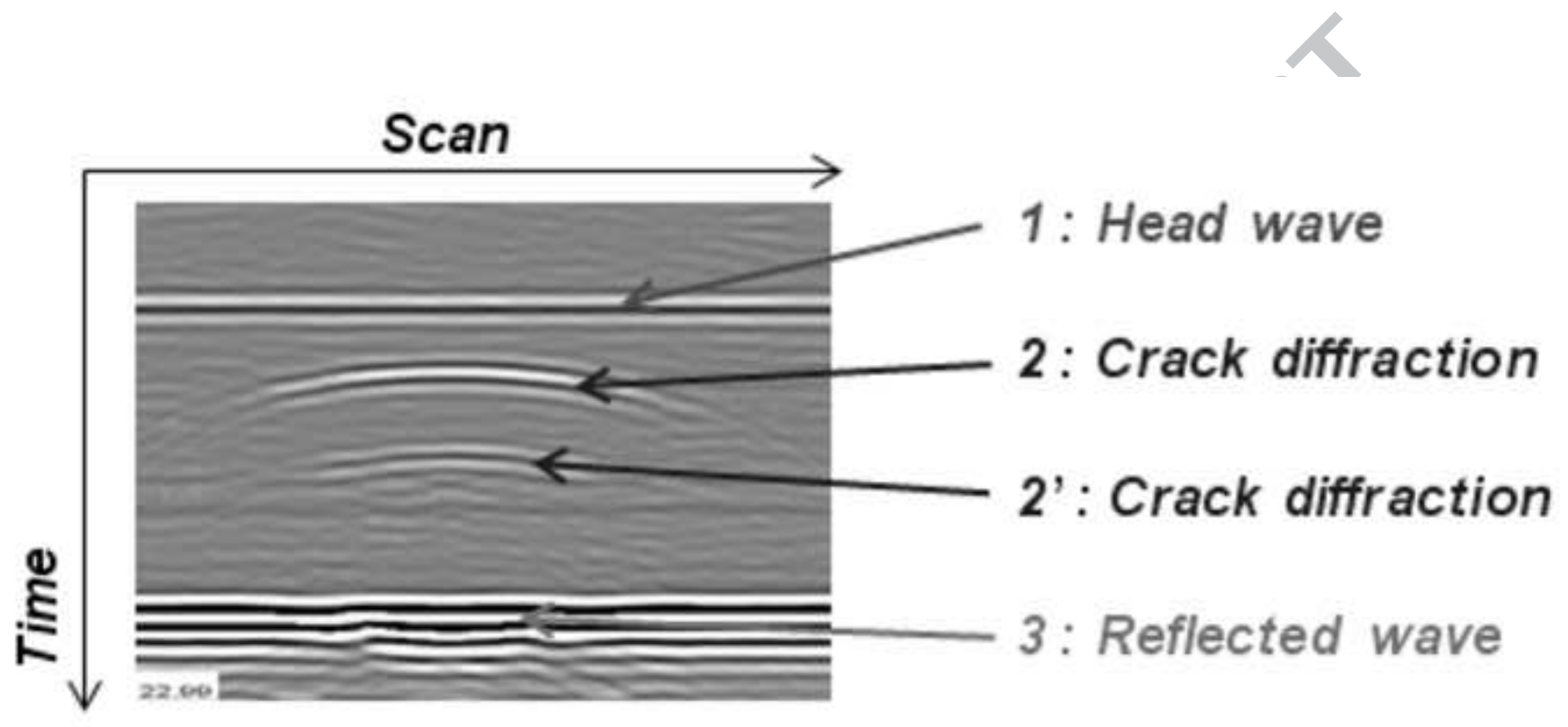

2: Crack diffraction

2': Crack diffraction

3: Reflected wave 
Removed part of the defect

\section{Remaining} matter

Remaining part of the defect

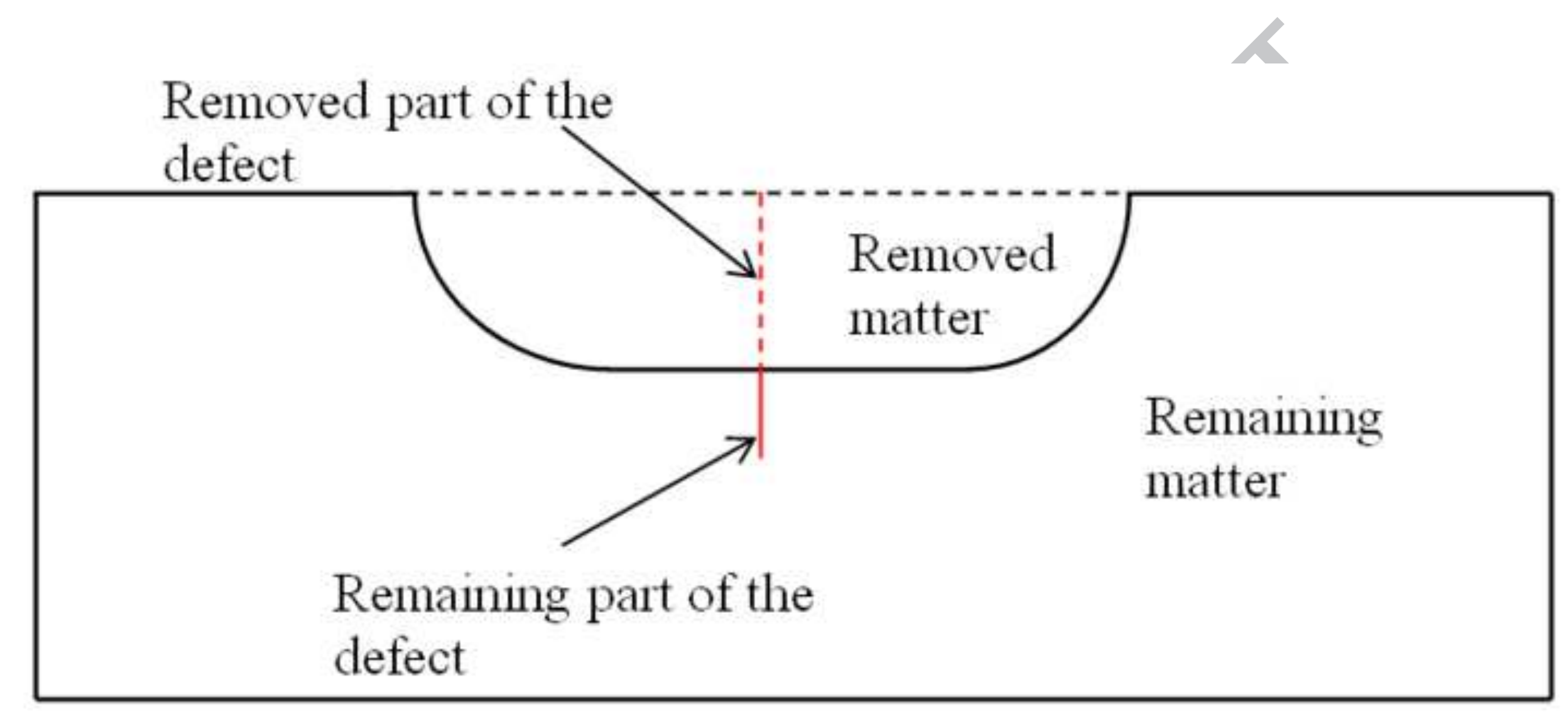

\section{Removed}

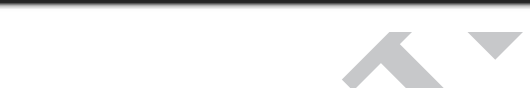


Removed part of the defect

\section{Remaining} matter

Remaining part of the defect

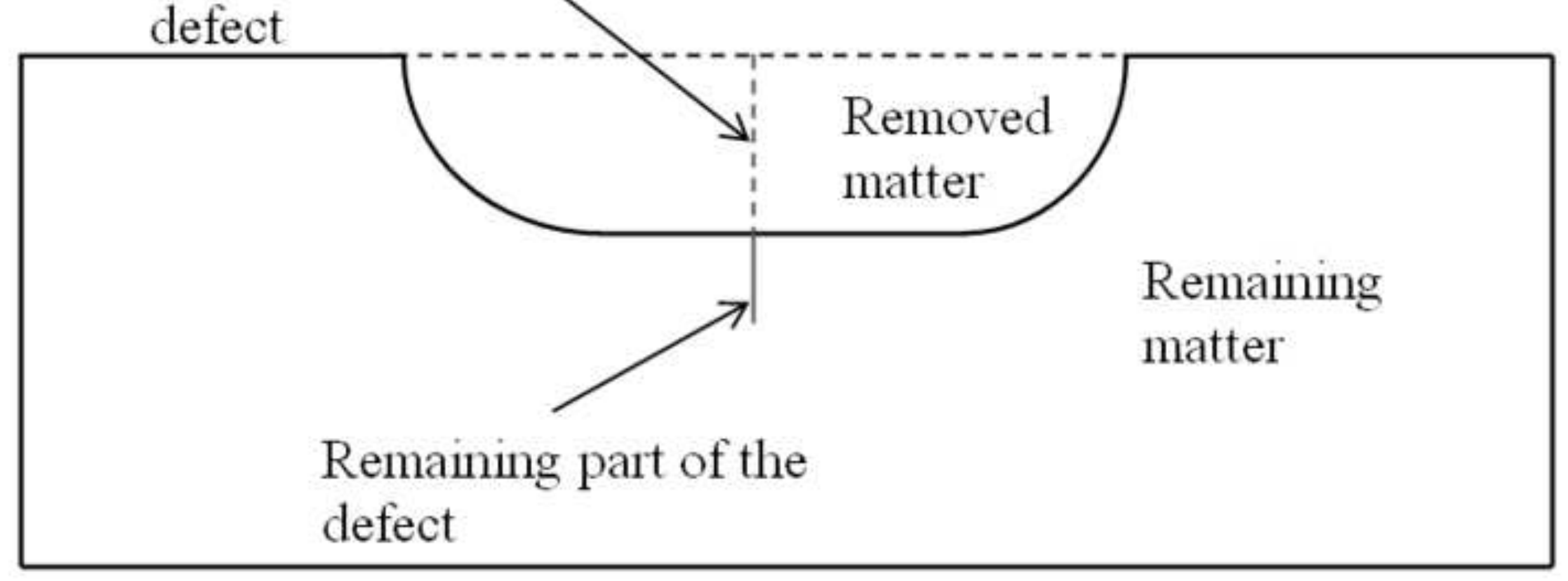




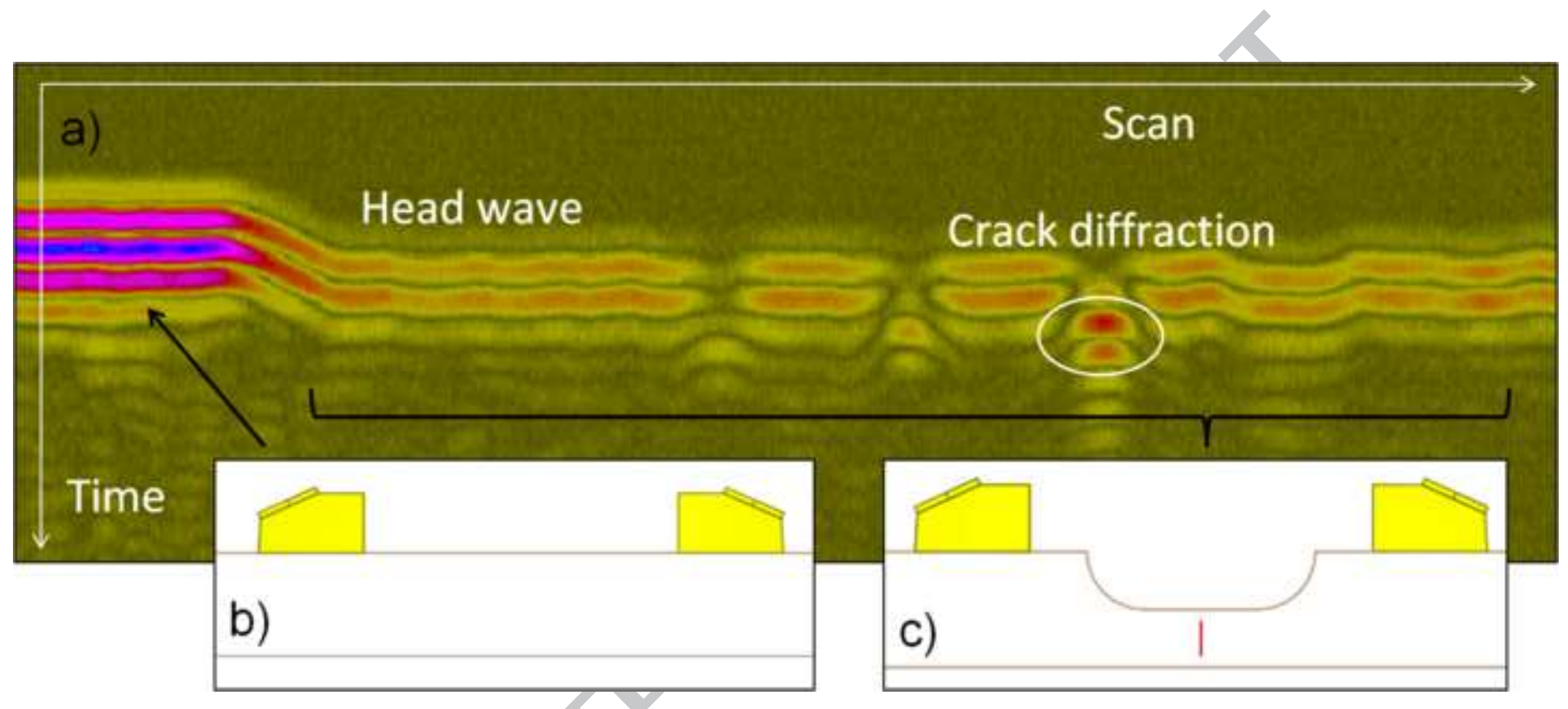

\section{Scan}

\section{Crack diffraction}

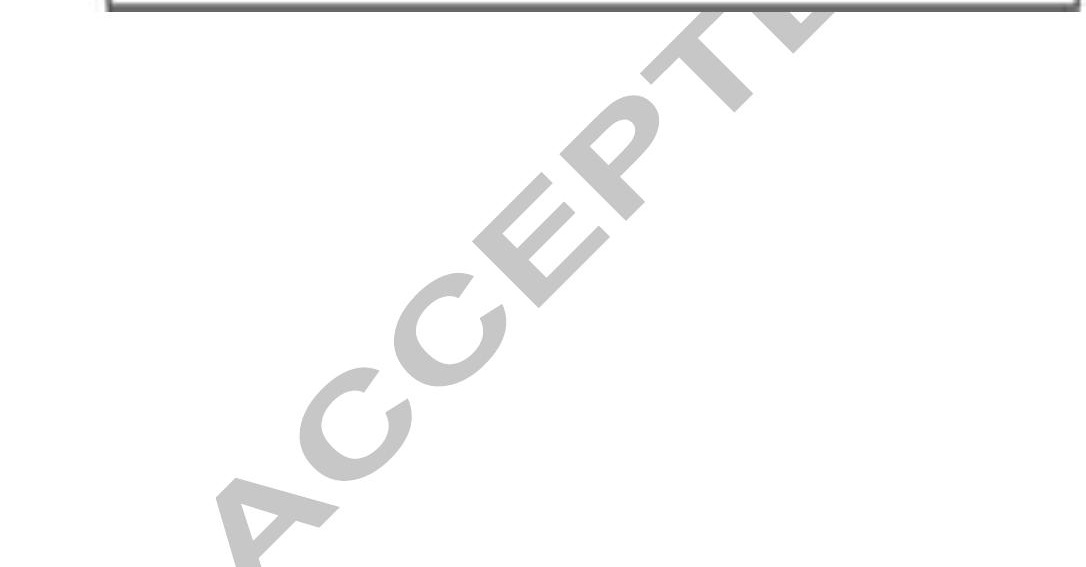

Head wave

Time 


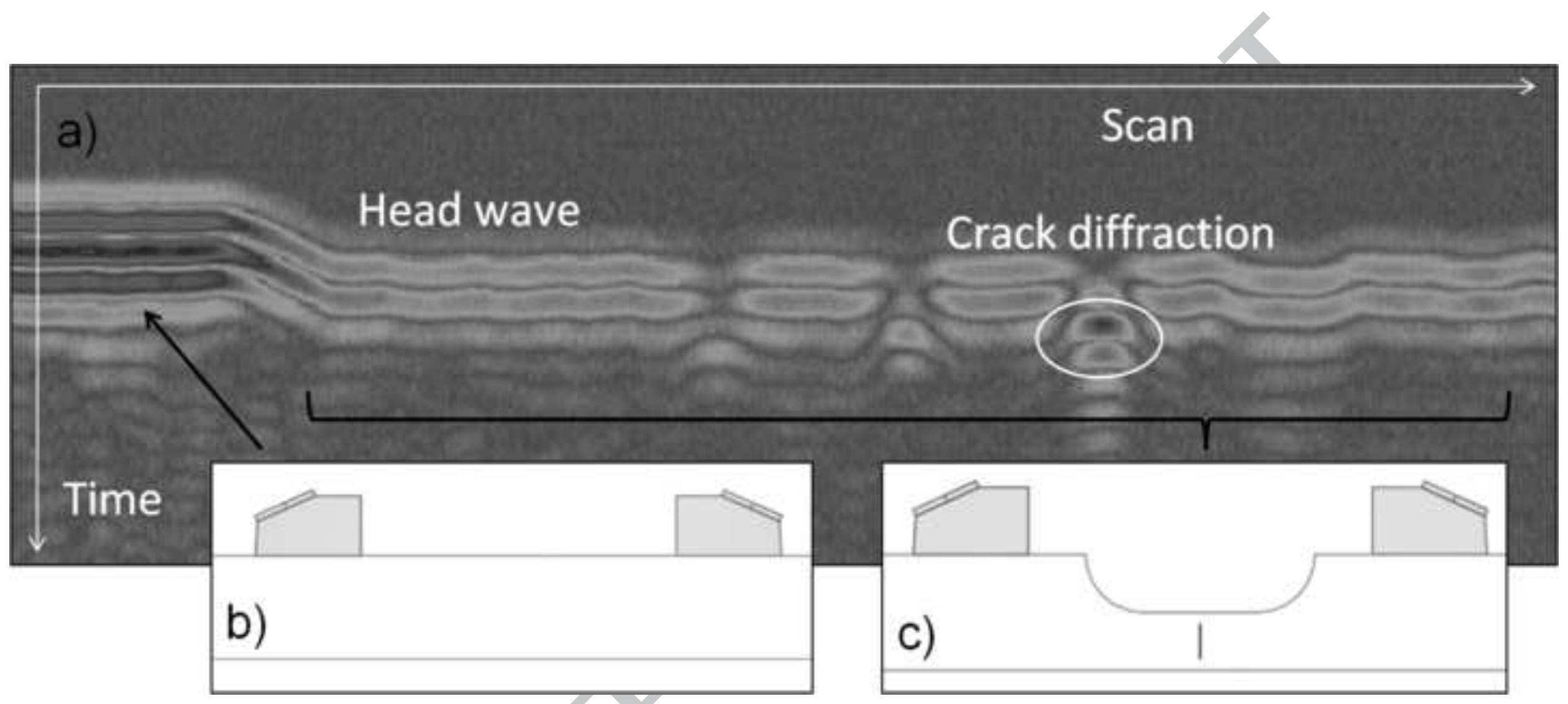



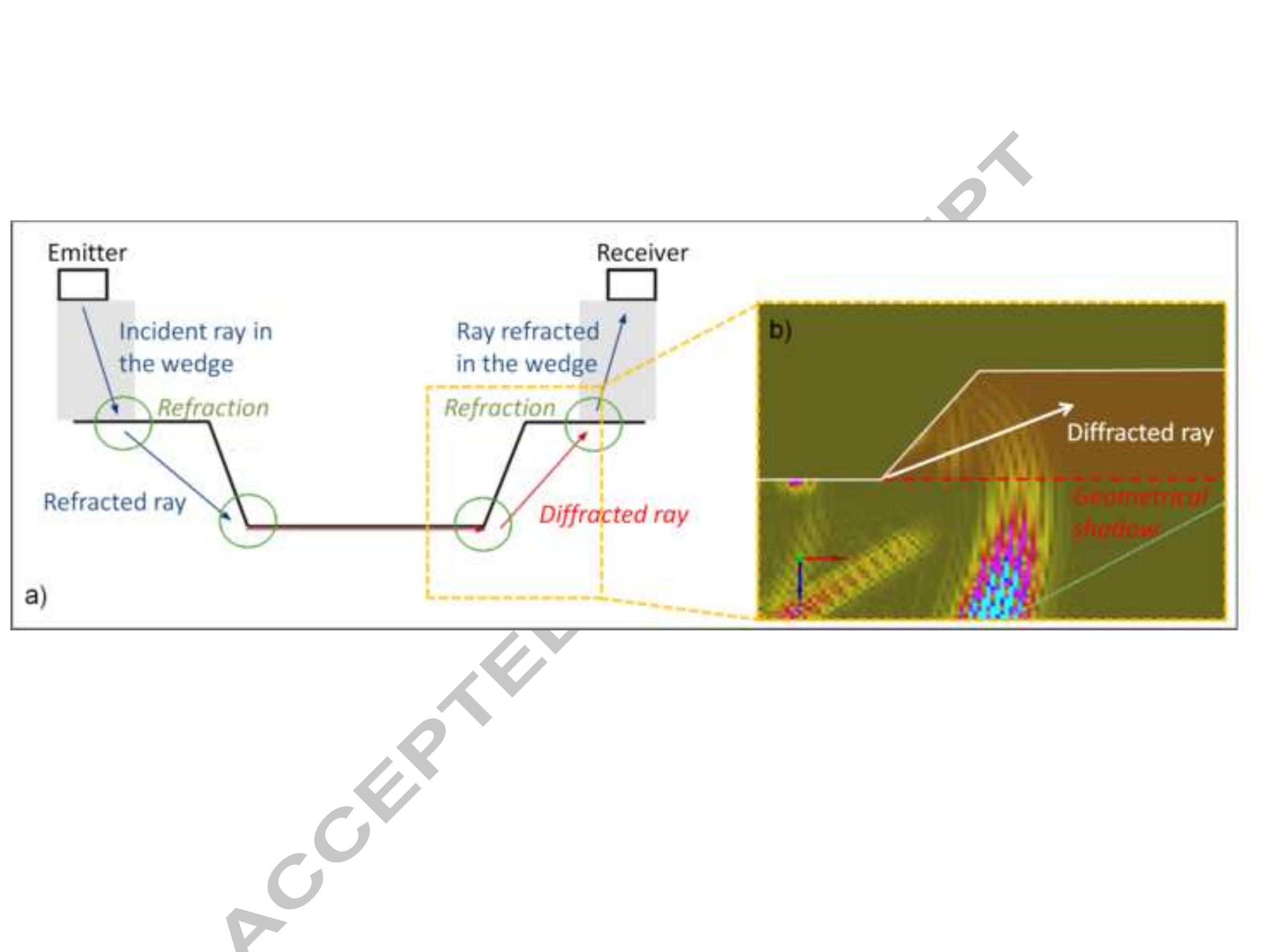


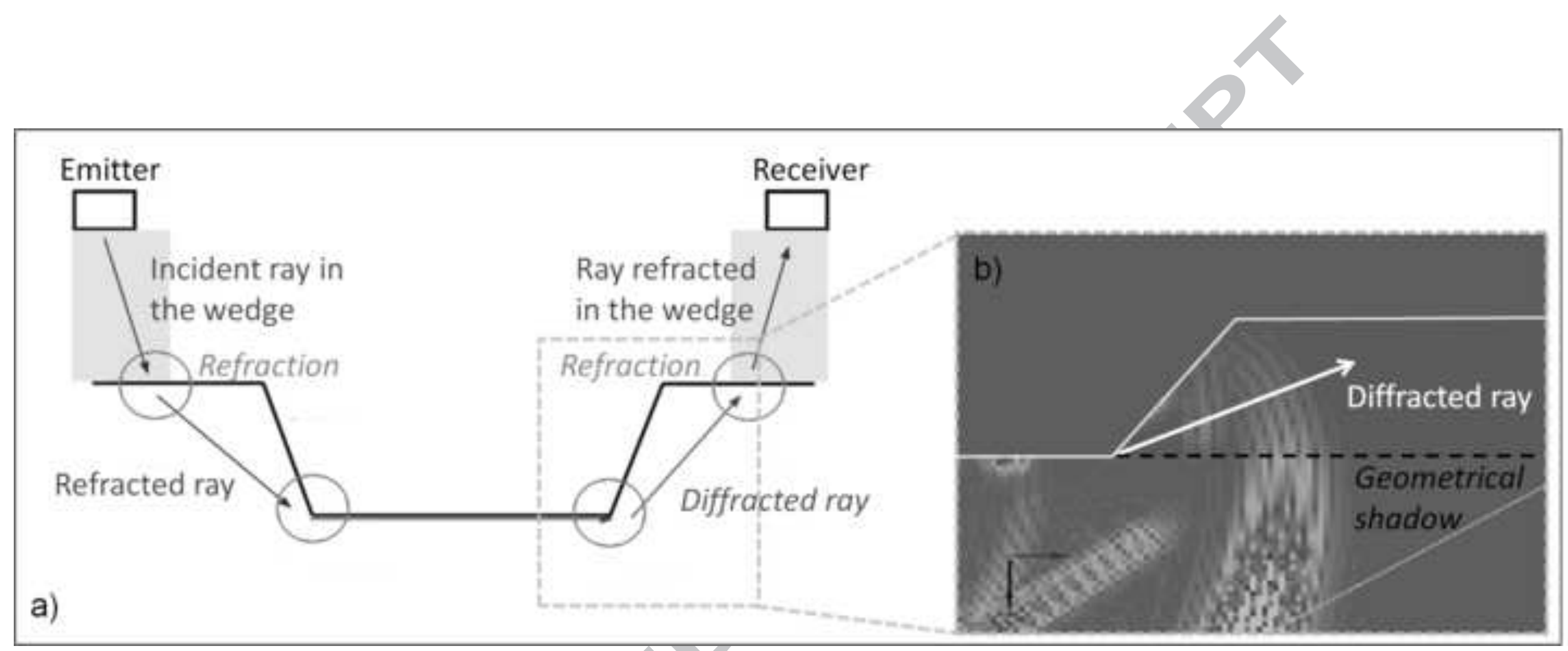


a)

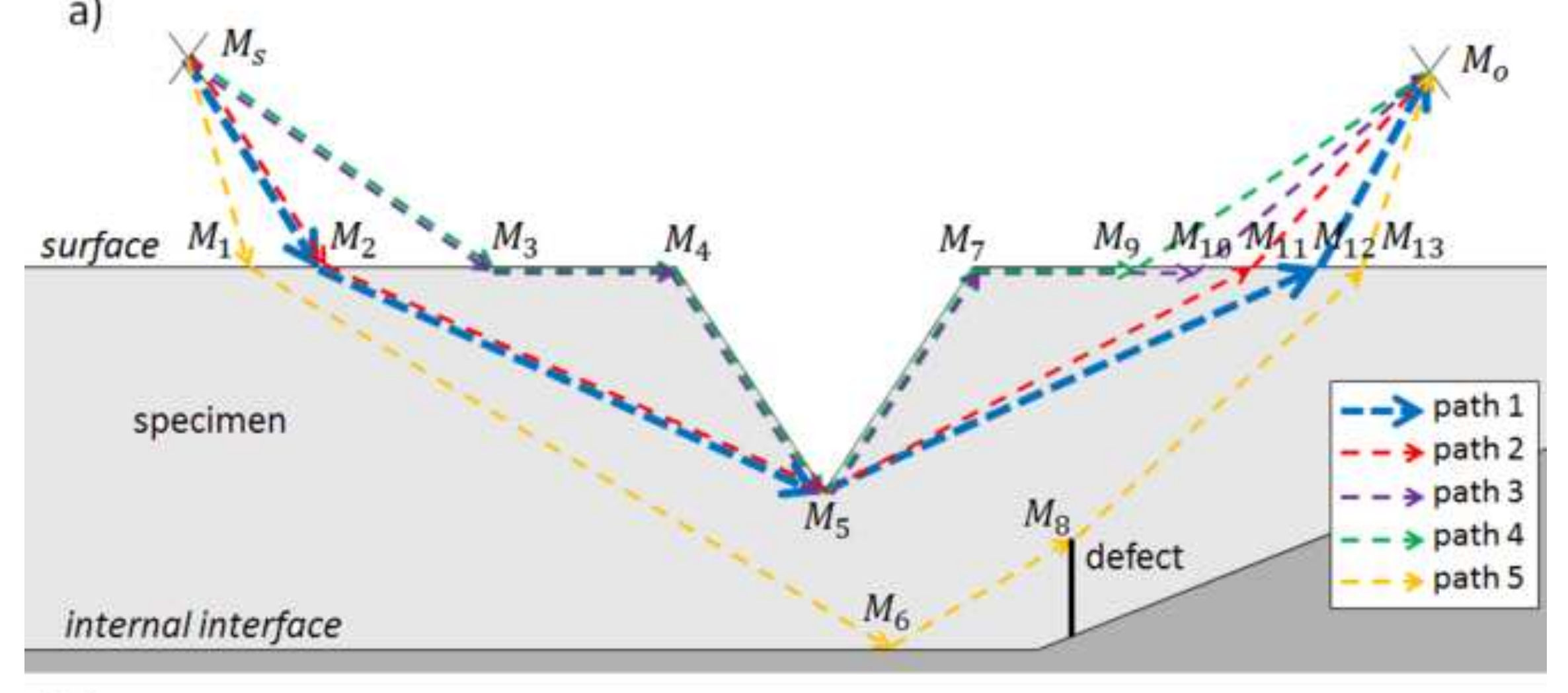

b)

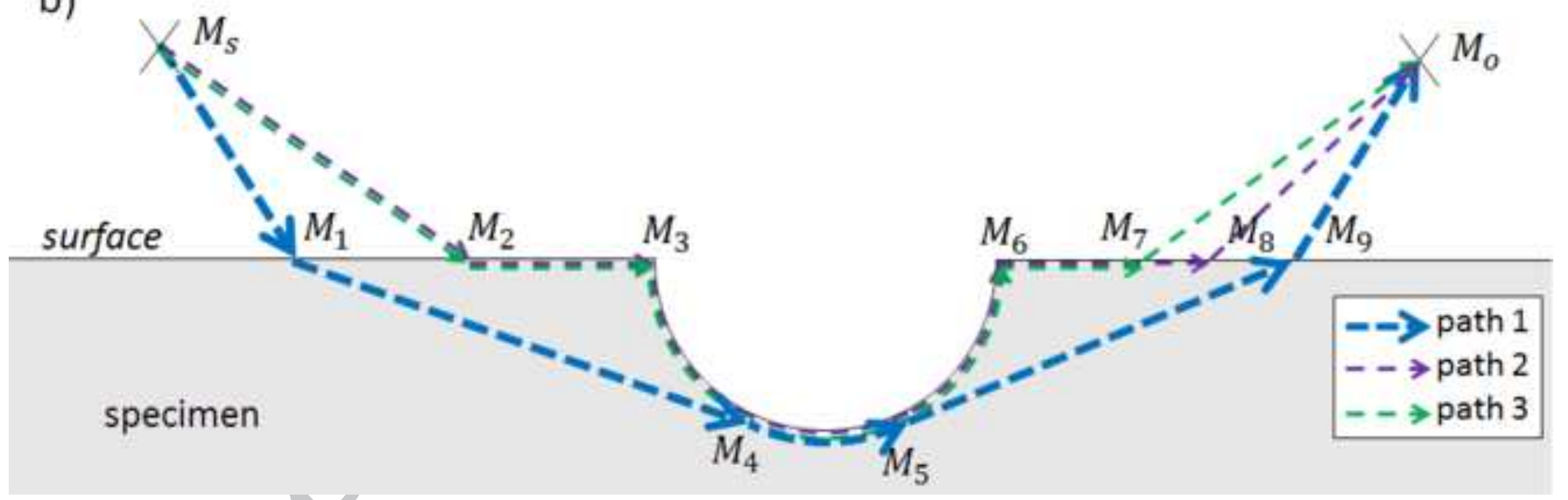

specimen 
a)

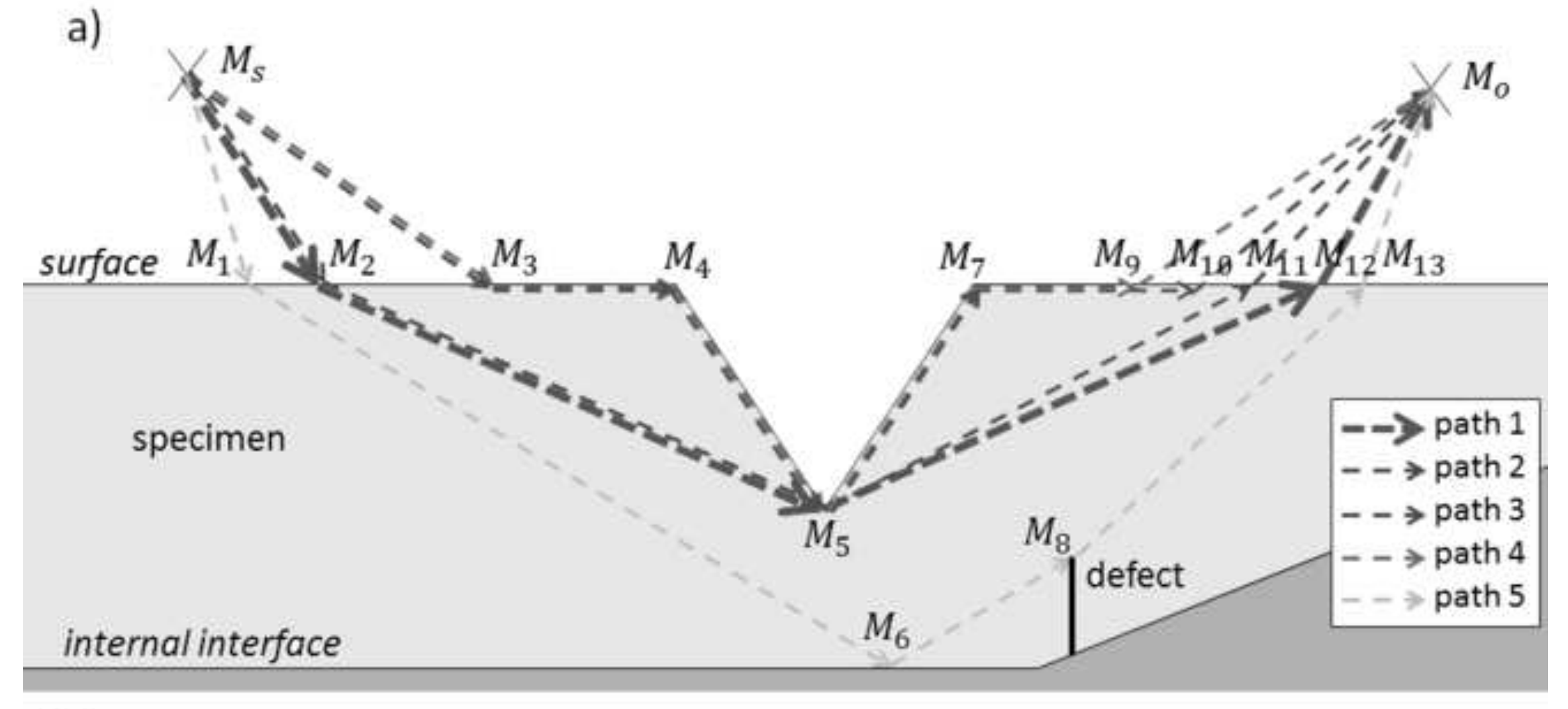

b)

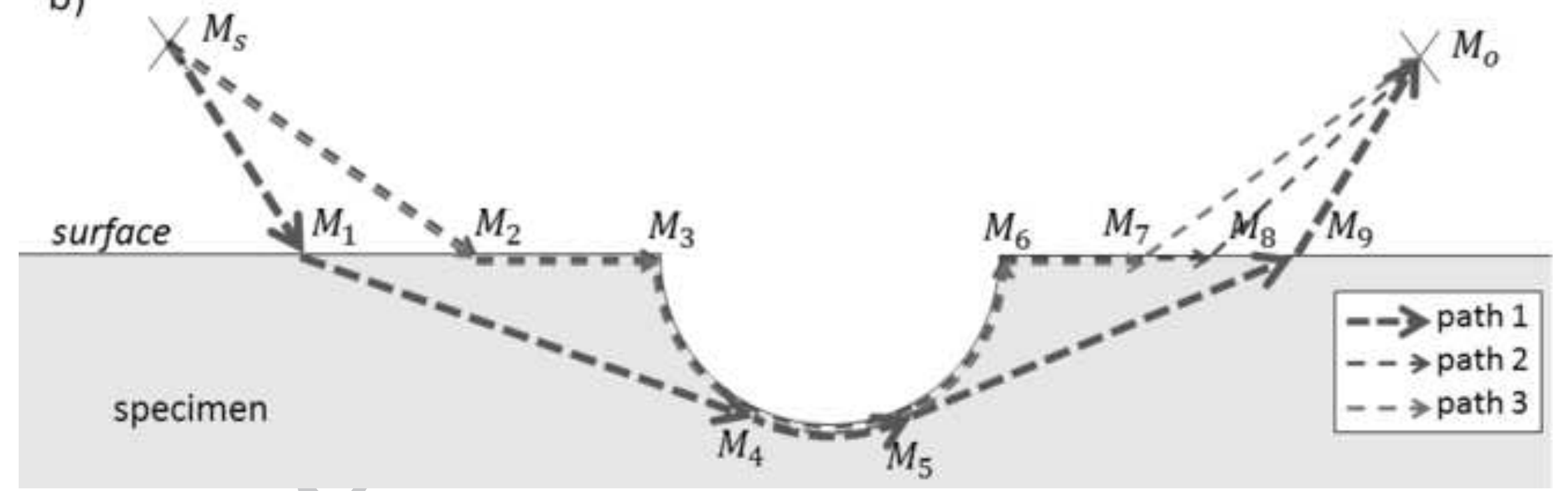 \\ ACCEPTED MANUSCRIPT}

specimen 


\section{ACCEPTED MANUSCRIPT}

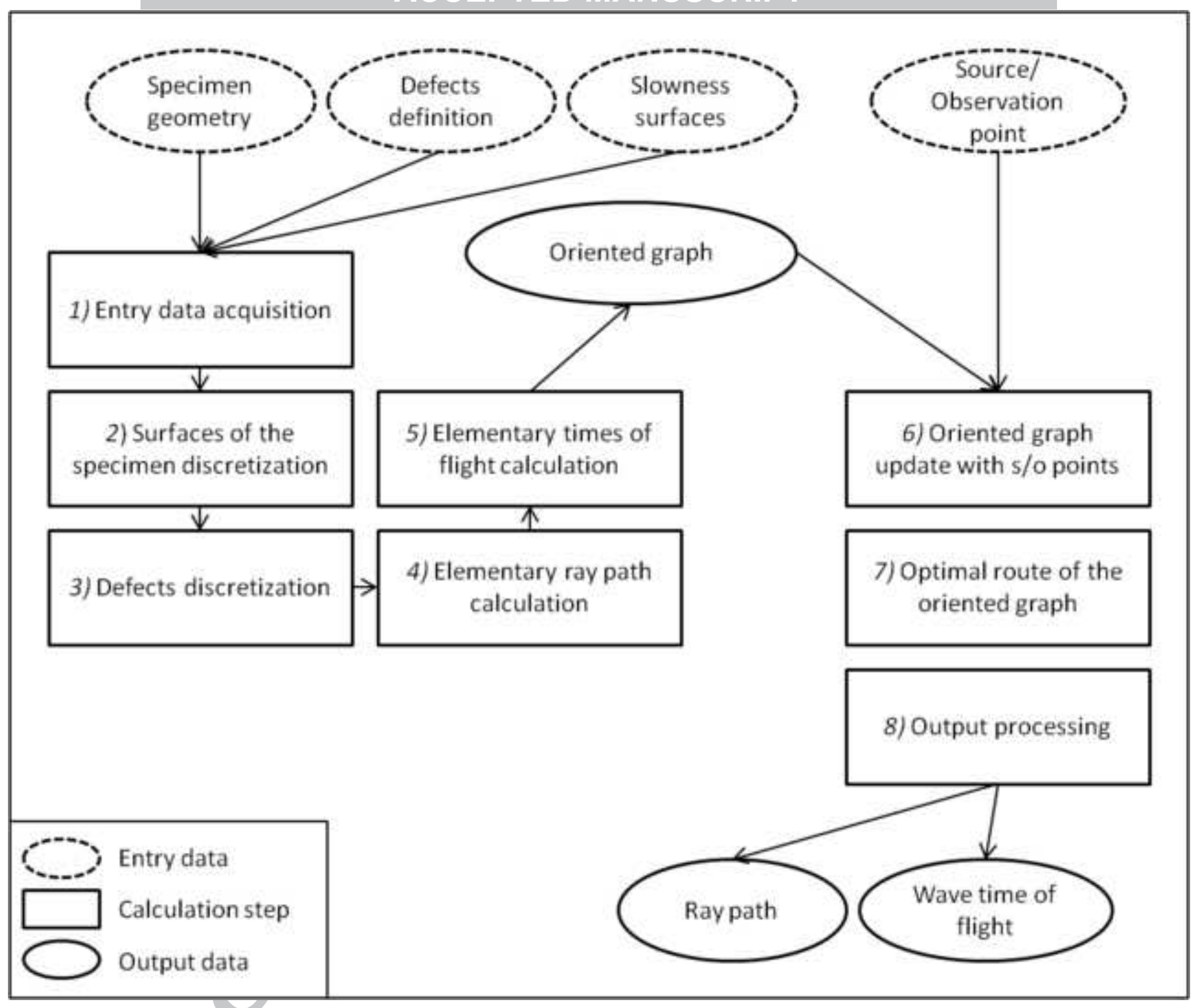




\section{ACCEPTED MANUSCRIPT}

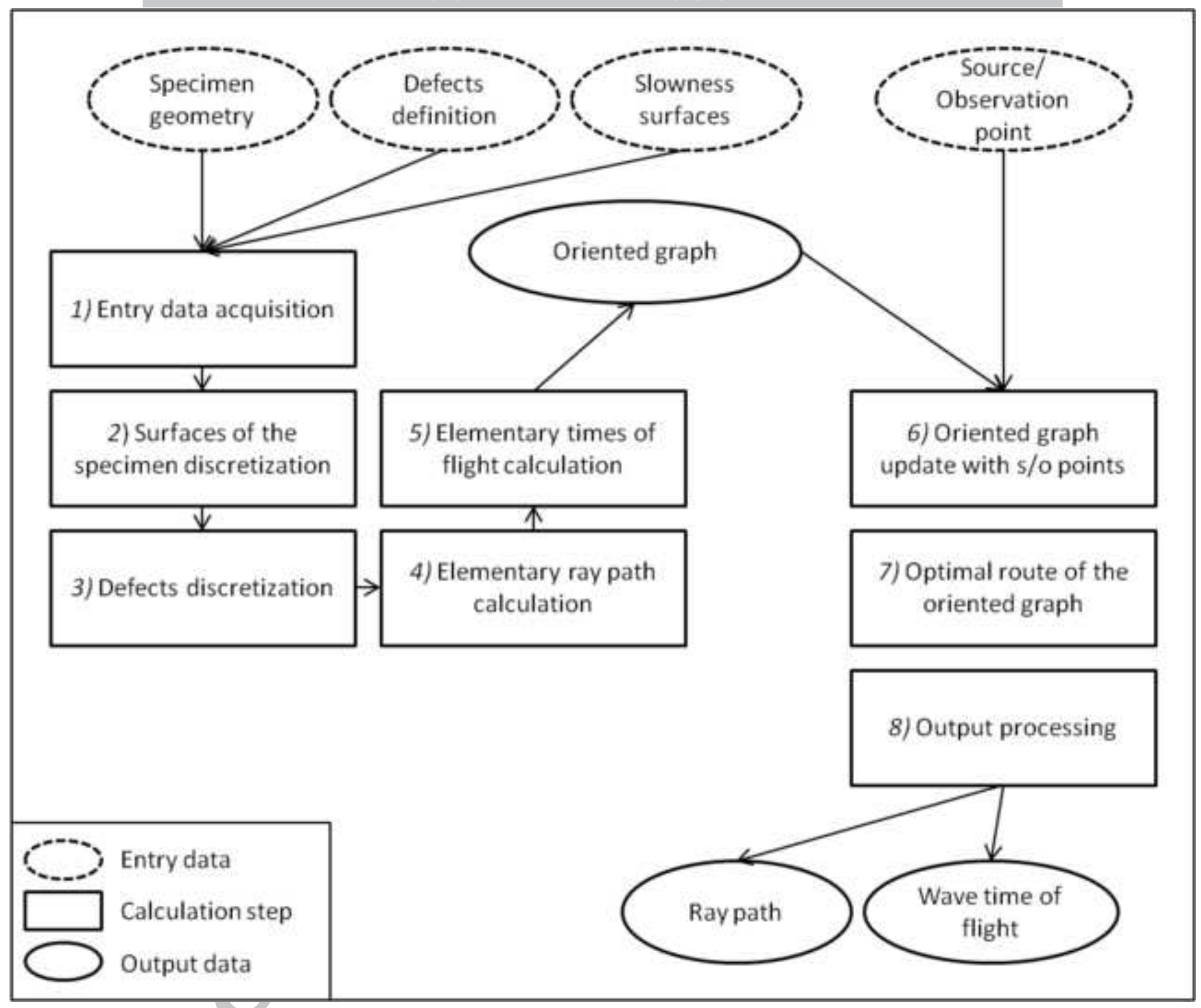




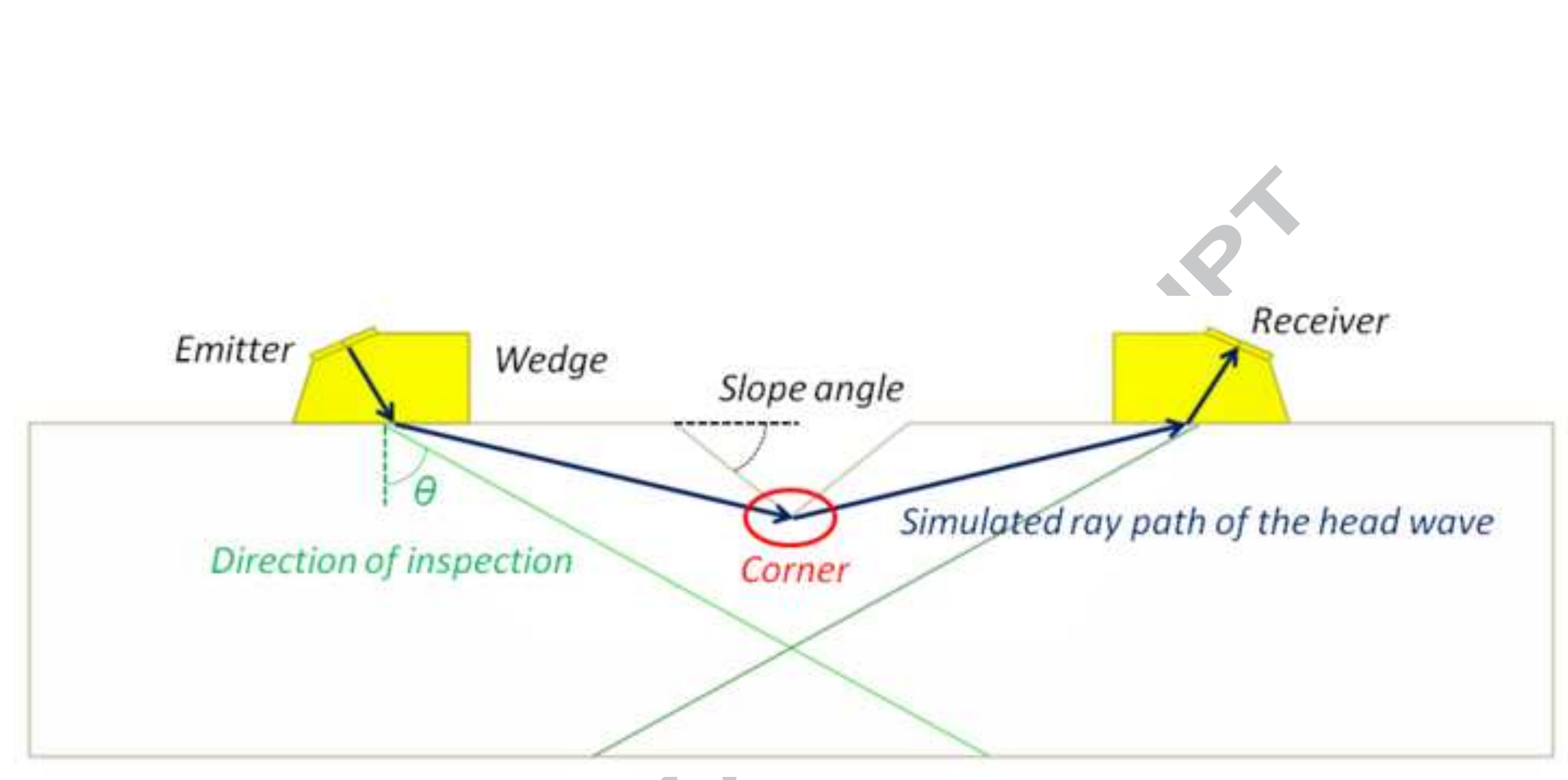




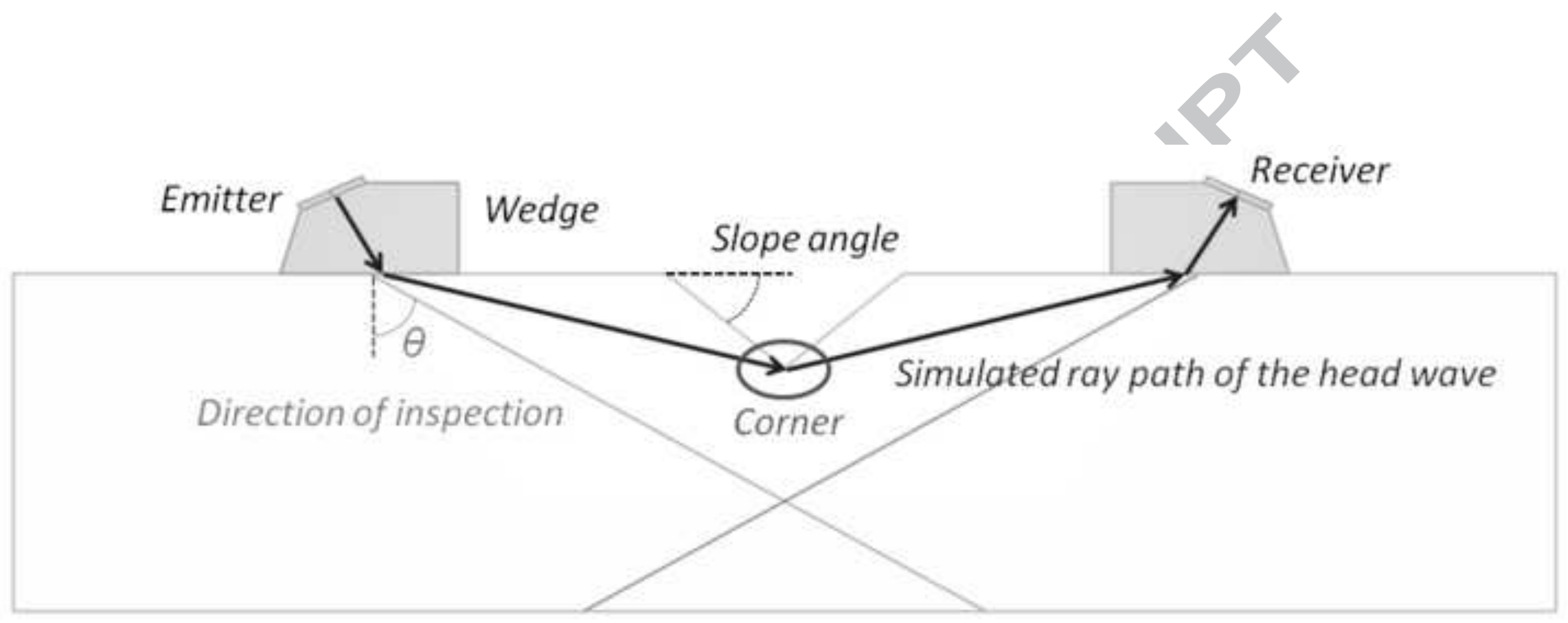




\section{ACCEPTED MANUSCRIPT}
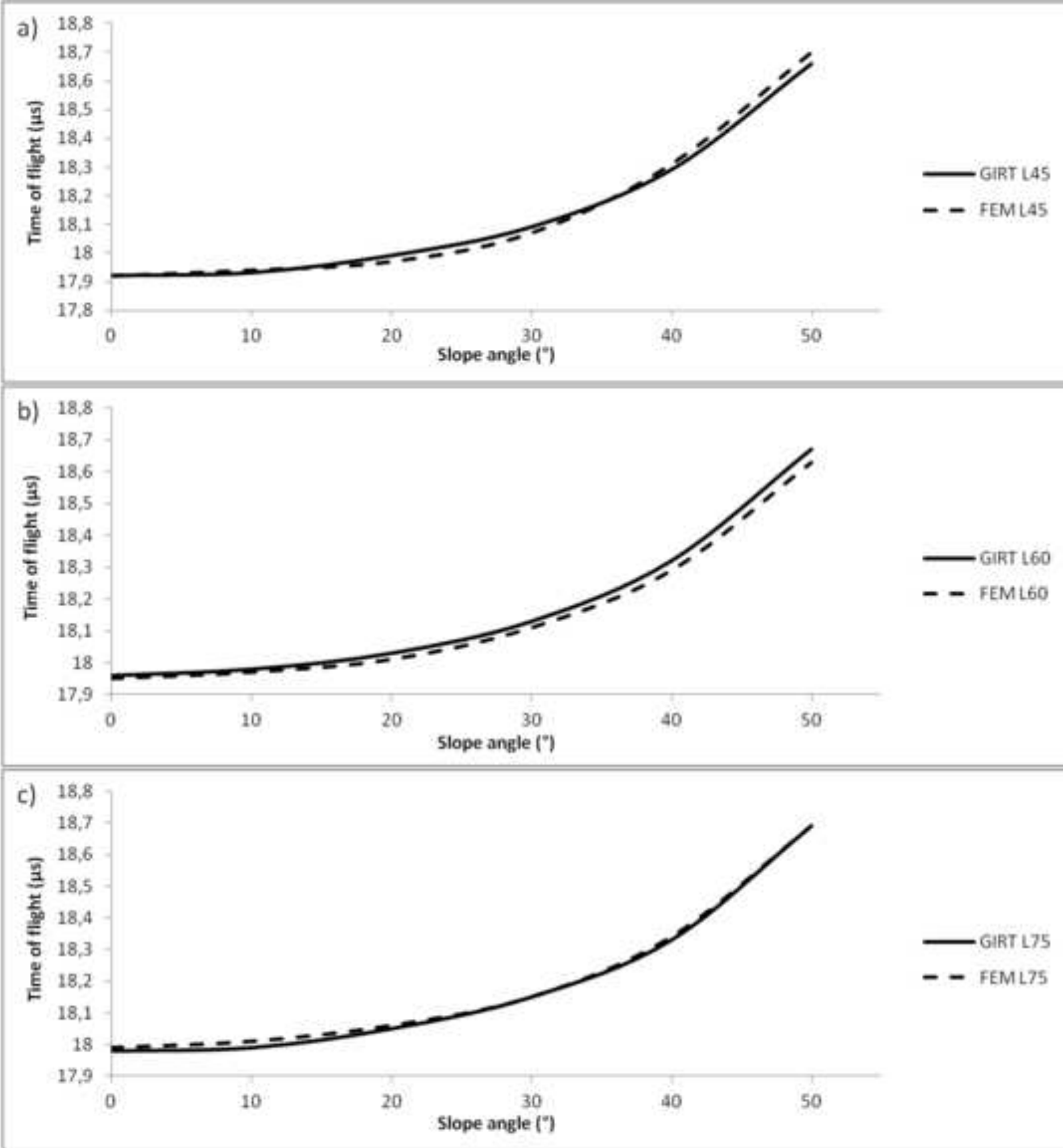

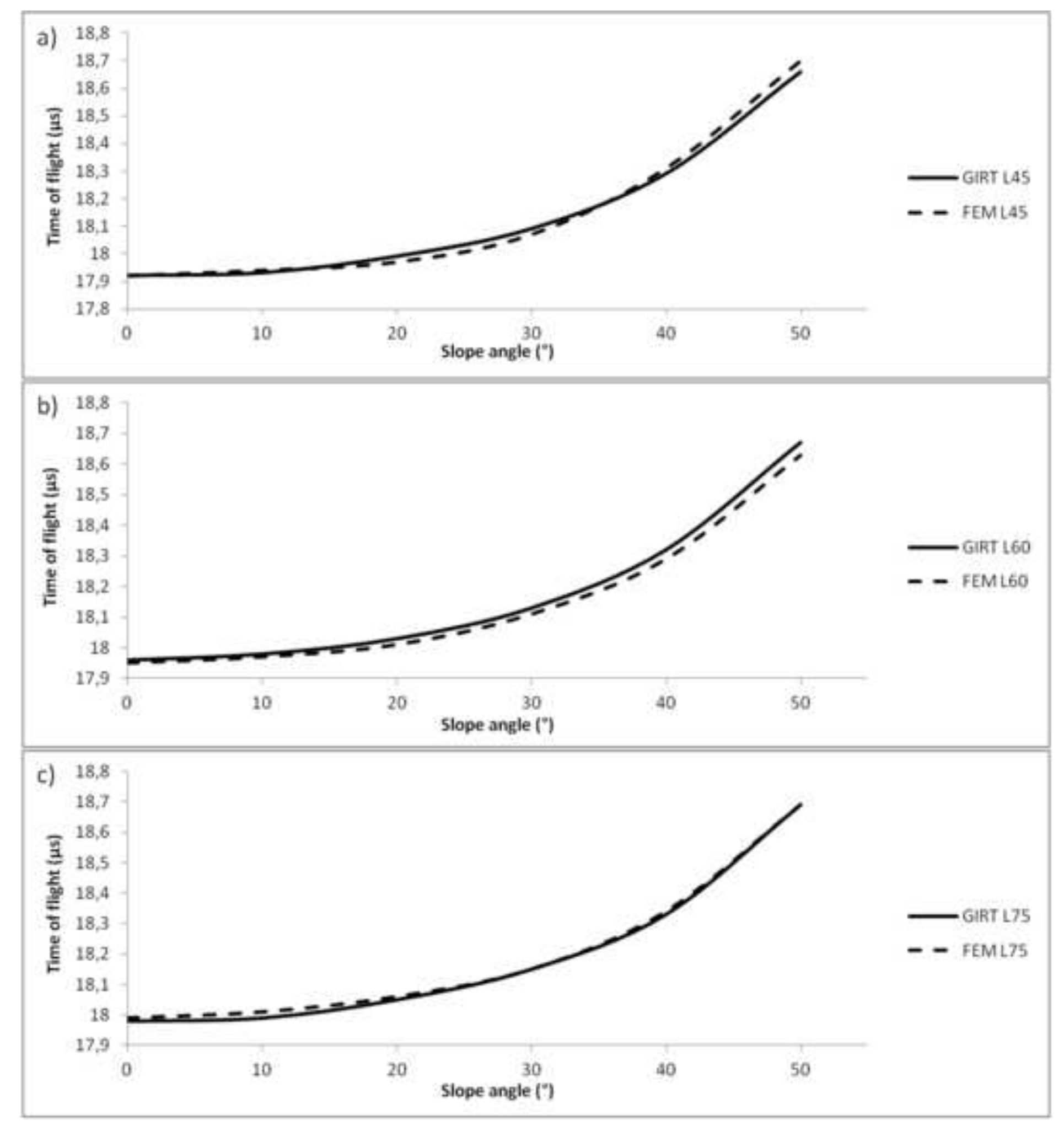

Figure 9 (b\&w)

\section{ACCEPTED MANUSCRIPT}
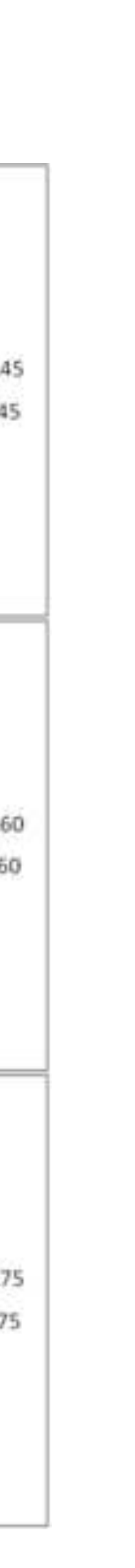

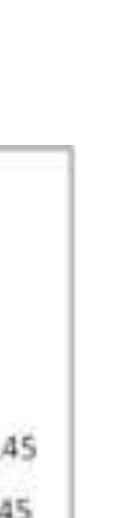
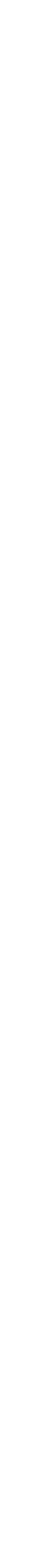


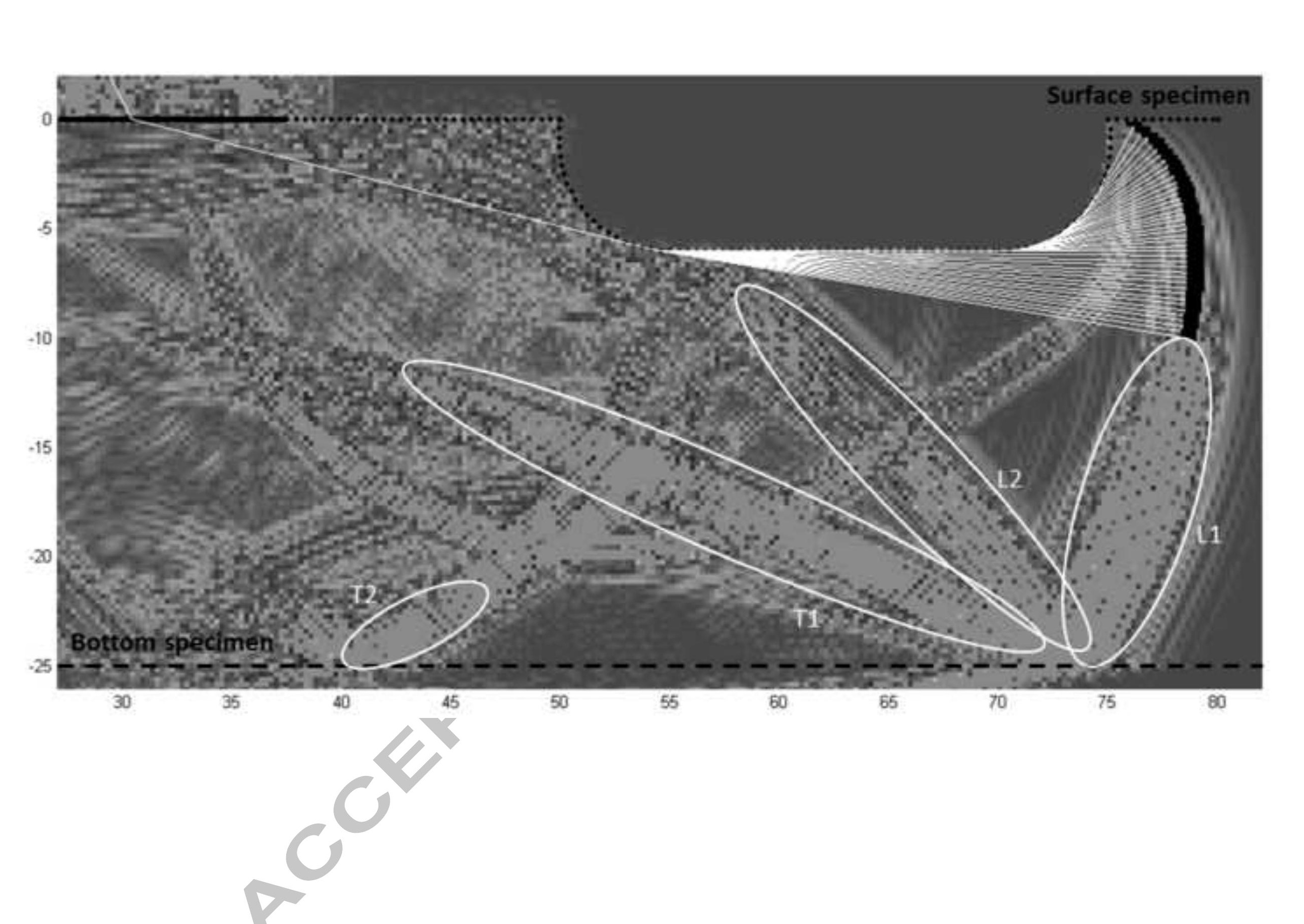



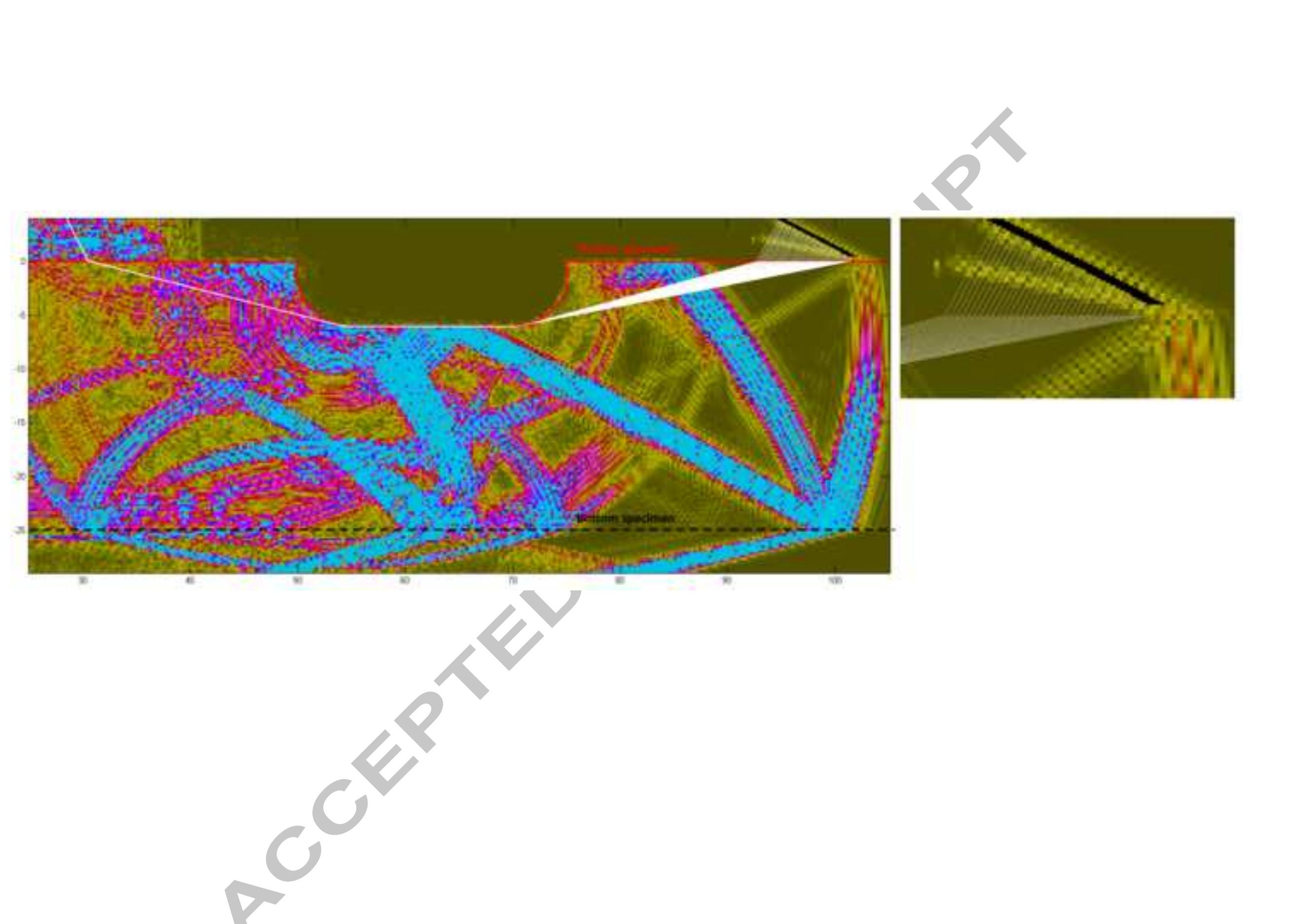

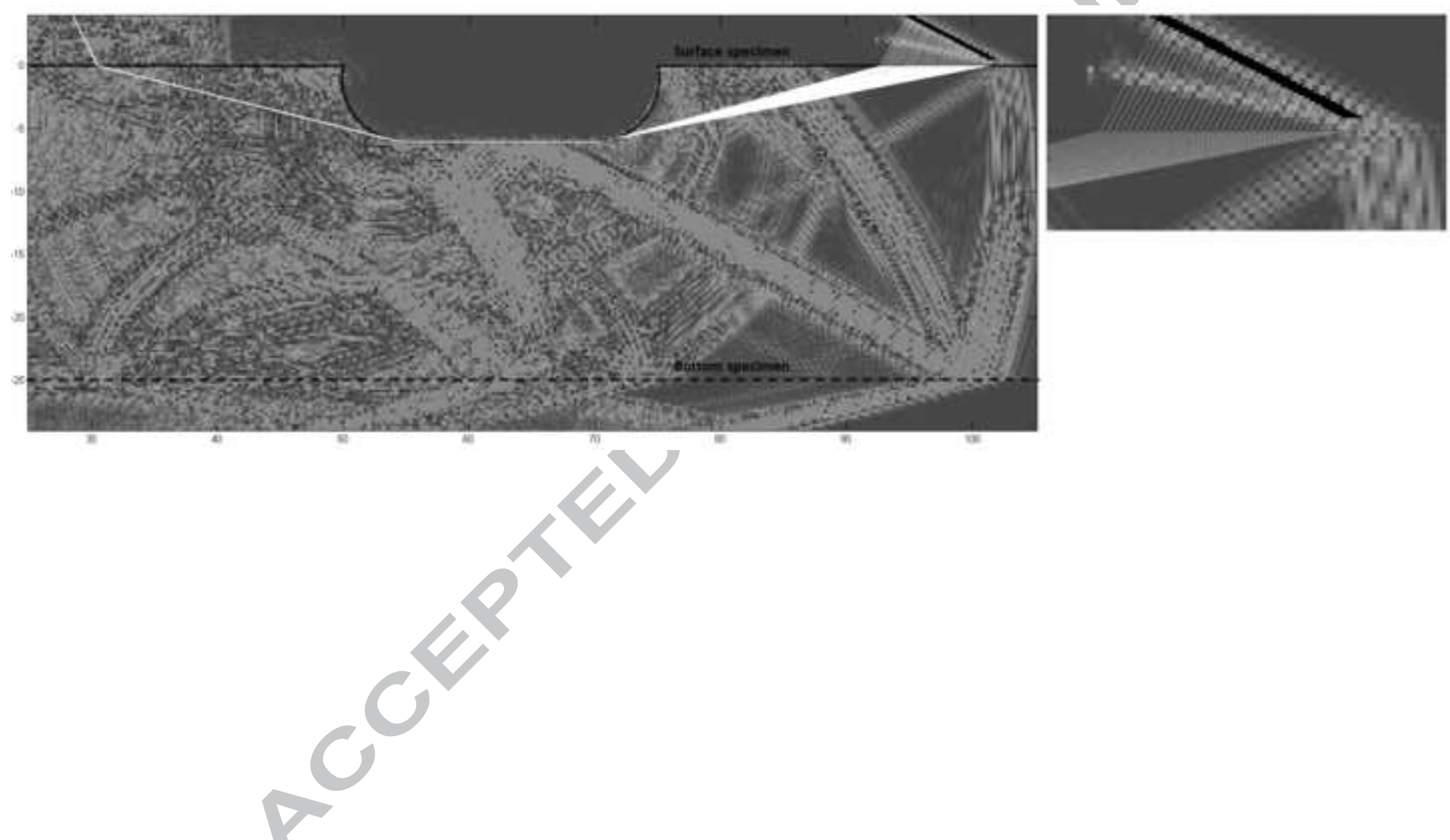

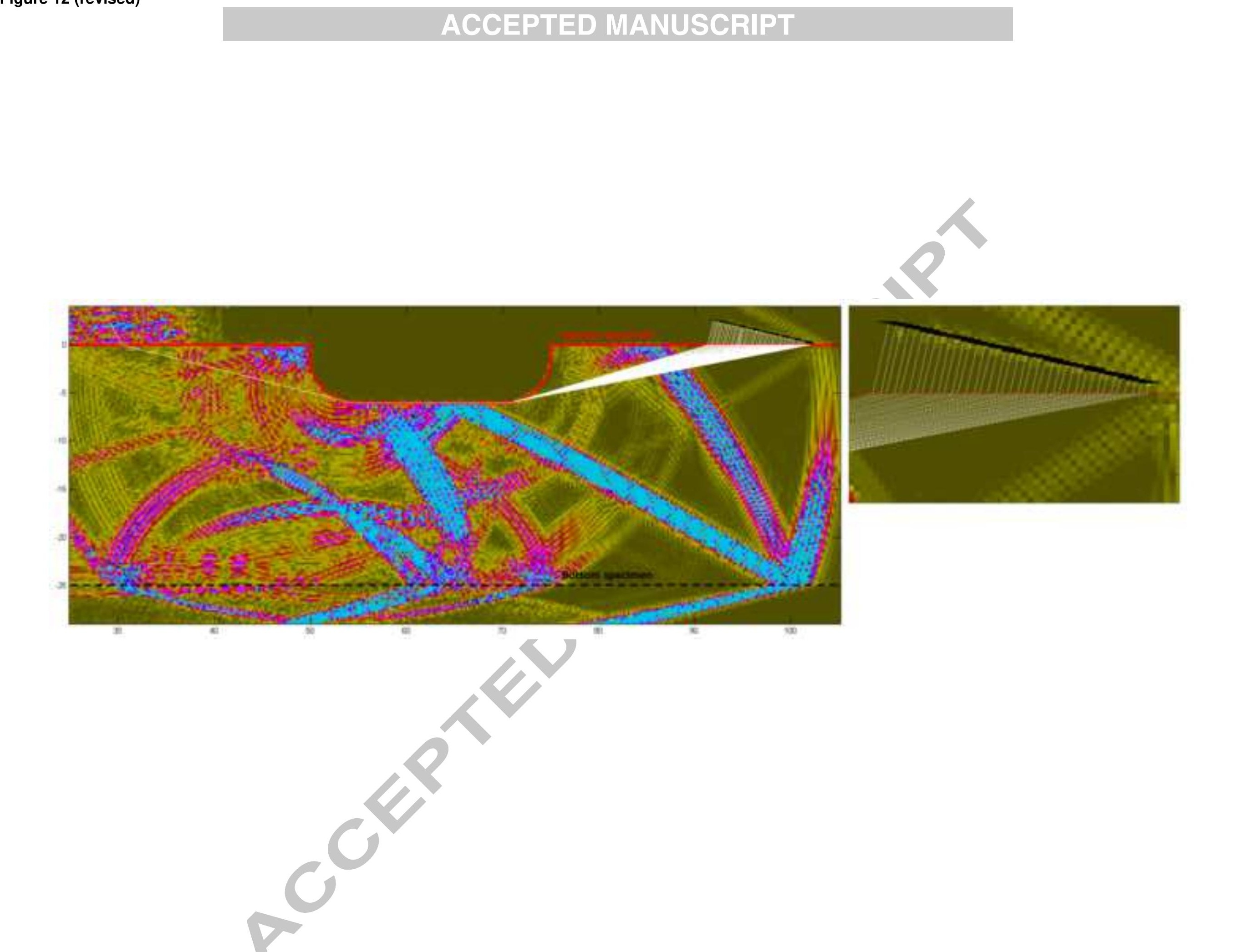

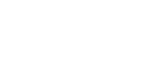
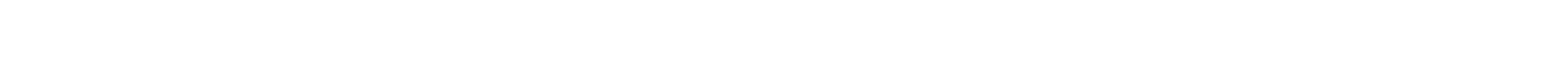

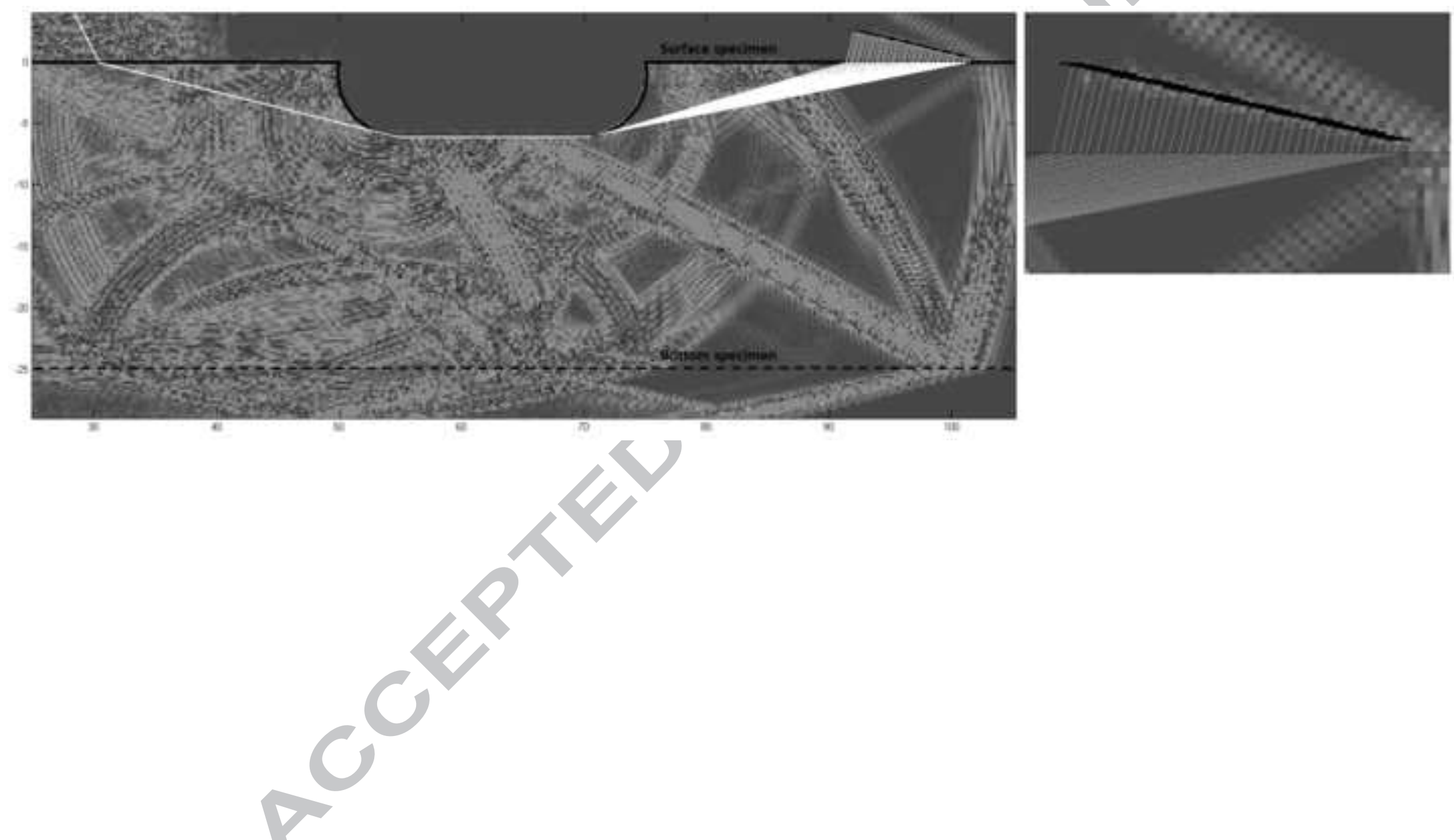

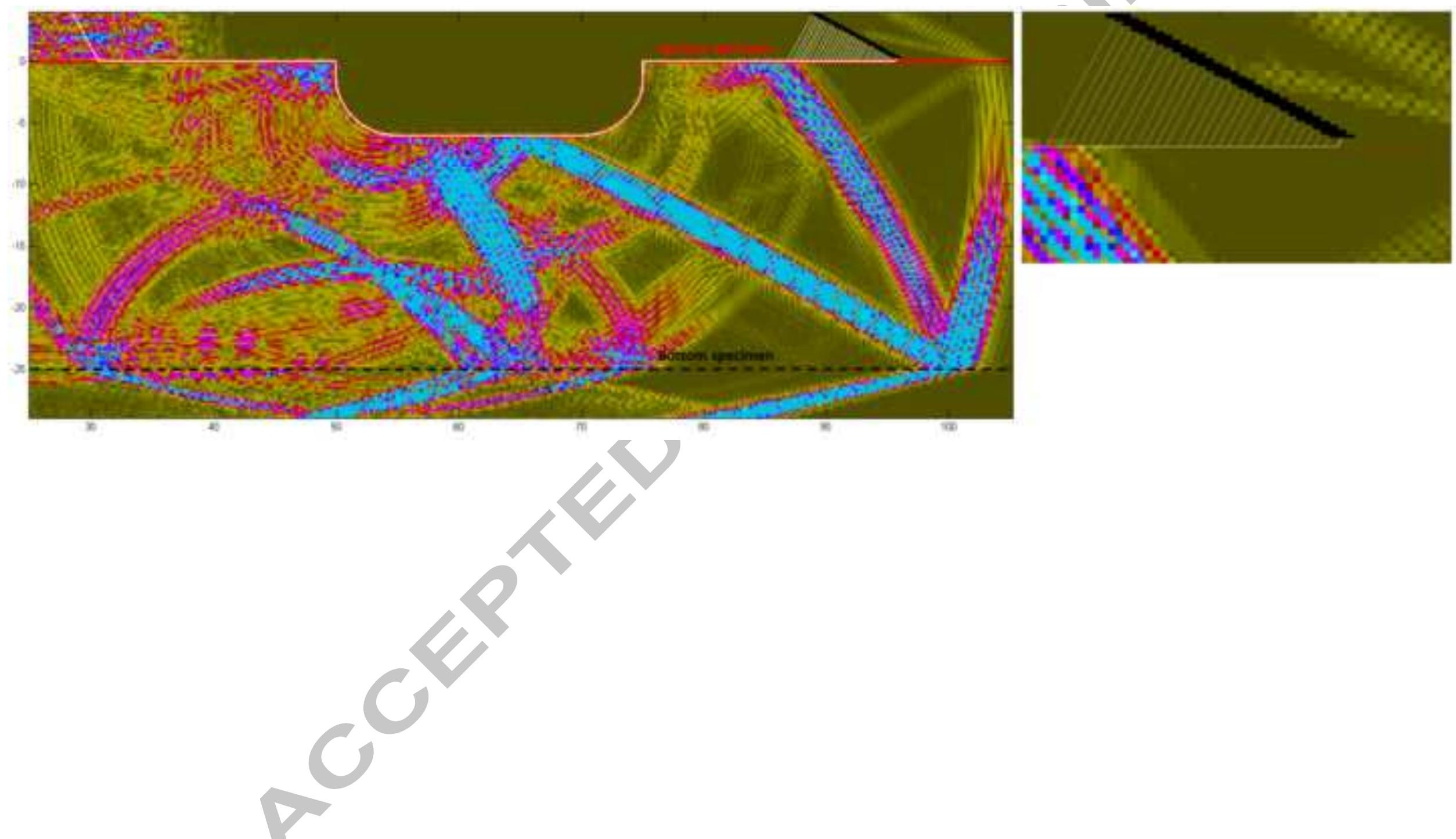

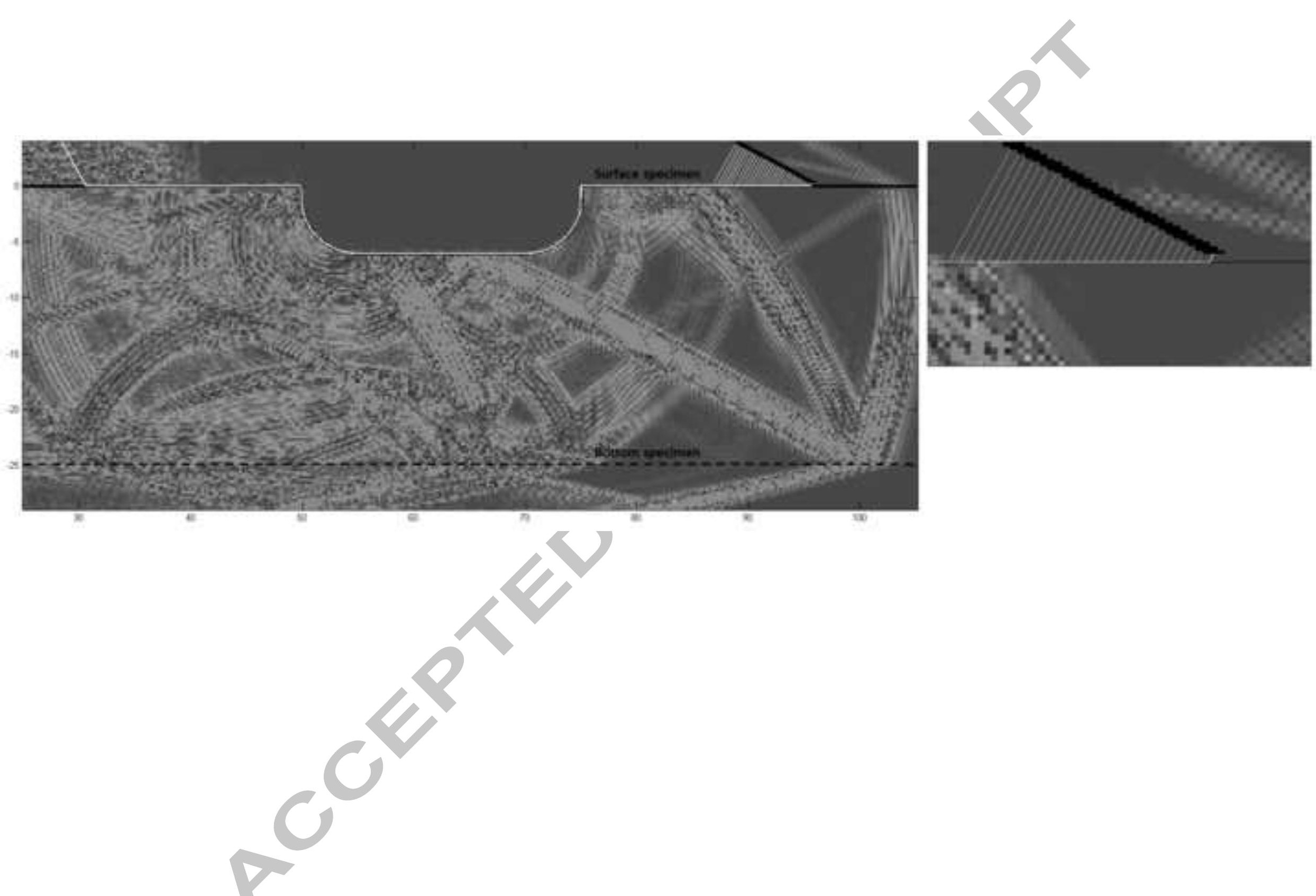
- This article provides a modeling study of head waves near irregular surfaces in NDT.

- Head wave propagation near such complex surfaces implies bulk mechanisms.

- A generic algorithm of ray tracing between interface points (GIRT) is developed.

- GIRT, based on Generalized Fermat's Principle, models effects of complex surfaces.

- The head wave fronts computed by GIRT are in good agreement with FEM simulations. 